\title{
Numerical Computation of Theta in a Jump-Diffusion Model by Integration by Parts
}

Delphine David — Nicolas Privault

\section{$\mathbf{N}^{\circ} \mathbf{5 8 2 9}$}

February, 82006

Thème NUM 



\title{
Numerical Computation of Theta in a Jump-Diffusion Model by Integration by Parts
}

\author{
Delphine David* , Nicolas Privault ${ }^{\dagger}$ \\ Thème NUM — Systèmes numériques \\ Projet MATHFI \\ Rapport de recherche $\mathrm{n}^{\circ} 5829$ - February, 82006 - 32 pages
}

\begin{abstract}
Using Malliavin weights in a jump-diffusion model we obtain an expression for Theta (the sensitivity of an option price with respect to the time remaining until exercise), with application to European and Asian options with non-smooth payoff function. In time inhomogeneous models our formula applies to the derivative with respect to the maturity date $T$, and its proof can be viewed as a generalization of Dupire's integration by parts to arbitrary payoff functions. In the time homogeneous case, our result applies to the derivative with respect to the current date $t$, but our representation formula differs from the one obtained from the Black-Scholes PDE in terms of Delta and Gamma. Optimal weights are computed by minimization of variance and numerical simulations are presented.
\end{abstract}

Key-words: Greeks, Theta, sensitivity analysis, jump-diffusion models, Malliavin calculus.

\footnotetext{
* Laboratoire de Mathématiques, Université de La Rochelle, 17042 La Rochelle Cedex, email: delphine.david@univ-lr.fr

† INRIA-Rocquencourt, email: nicolas.privault@inria.fr
} 


\section{Calcul numérique de Theta par intégration par parties dans un modèle de diffusion avec sauts}

Résumé : Par le calcul de Malliavin nous obtenons une expression de Theta (la sensibilité d'un prix d'option par rapport au temps restant jusqu'à l'exercice), avec application aux options européennes et asiatiques à fonction de payoff irrégulière. Dans le cas non-homogène en temps, notre formule s'applique à la dérivée par rapport à la date d'exercice $T$, et sa preuve peut être vue comme une généralisation de l'argument de Dupire à des fonctions de payoff arbitraires. Dans le cas homogène en temps, notre résultat s'applique à la dérivée par rapport à la date courante $t$, mais notre formule de représentation diffère de celle obtenue par l'EDP de Black-Scholes en termes de Delta et Gamma. Nous déterminons également des poids de variance minimale, et des simulations numériques sont présentées.

Mots-clés : Grecs, Theta, analyse de sensibilité, diffusions avec sauts, calcul de Malliavin. 


\section{Introduction}

Predicting the variations of option prices can be a difficult task, and in order to make such predictions it is important to understand which factors contribute to the movement of prices, and with what effect. The Delta, Gamma, Vega, Rho and Theta of an option position, collectively known as the "Greeks", provide a way to measure the sensitivity of an option prices to factors such as spot price, volatility, interest rate and time respectively.

Sensitivity analysis using the Malliavin calculus in finance has been developed by several authors, starting with [7]. In this paper we aim at applying similar methods to the computation of Theta, which measures the variations of an option price with respect to the time until exercise, while other parameters remain constant. Our argument consists in a combination of Itô calculus with integration by parts on the Wiener space. Let

$$
C(x, t, T)=e^{-\int_{t}^{T} r_{s} d s} \mathbb{E}\left[\phi\left(S_{T}\right) \mid S_{t}=x\right]
$$

denote the price at time $t$ of a European type option with spot price $x$, interest rate and volatility functions $r_{t}, \sigma_{t}(\cdot)$, maturity $T$, strike price $K$, payoff function $\phi$, and price process $\left(S_{t}\right)_{t \in[0, T]}$. In this general setting, two versions of Theta can be computed, namely

$$
\text { Theta }_{t}=\frac{\partial C}{\partial t}(x, t, T), \quad \text { and } \quad \operatorname{Theta}_{T}=\frac{\partial C}{\partial T}(x, t, T) .
$$

Theta $_{t}$ is used for European options for which $T$ is a fixed date, whereas Theta $T$ can be used in case $T$ is a free parameter, e.g. for the choice of the exercise date of a European option, or for American type contracts.

As is well-known, Theta $t_{t}$ is involved in the Black-Scholes PDE: for example, in a continuous market with interest rate and volatility functions $r_{t}, \sigma_{t}(\cdot)$ we have

$$
\text { Theta }_{t}=r_{t} C(x, t, T)-x r_{t} \frac{\partial C}{\partial x}(x, t, T)-\frac{1}{2} x^{2} \sigma_{t}^{2}(x) \frac{\partial^{2} C}{\partial x^{2}}(x, t, T) .
$$

In case the Markov process $\left(S_{t}\right)_{t \in[0, T]}$ is time homogeneous, i.e. when the coefficients $r, \sigma(\cdot)$ are time independent, the price $C$ satisfies

$$
C(x, t, T)=e^{-(T-t) r} \mathbb{E}\left[\phi\left(S_{T-t}\right)\right]
$$

$\mathrm{RR} \mathrm{n}^{\circ} 5829$ 
and becomes a function of the remaining time $\tau:=T-t$ until exercise. In this case we have

$$
\text { Theta }_{T}=- \text { Theta }_{t}=\frac{\partial C}{\partial \tau}(x, t, t+\tau),
$$

which will be simply denoted by Theta.

In this paper we compute Theta $T$ in a time inhomogeneous setting, using the Itô formula. In the homogeneous case this yields a representation formula which differs from the one given for Theta $t=-$ Theta $_{T}$ by the Black-Scholes PDE (1.1) in terms of the Greeks Delta $\frac{\partial C}{\partial x}$ and Gamma $\frac{\partial^{2} C}{\partial x^{2}}$, and relies on a single weight whose variance is minimized individually. Since our approach is directly based on time derivatives, it does not require the use of the first and second variation processes which would be normally needed in the computation of Gamma by integration by parts. In the particular case of European options in a continuous market, with price

$$
C(x, t, T, K)=e^{-\int_{t}^{T} r_{s} d s} \mathbb{E}\left[\left(S_{T}-K\right)^{+} \mid S_{t}=x\right],
$$

our method extends the argument of [6] which yields the Dupire PDE

$$
\operatorname{Theta}_{T}=-K r_{T} \frac{\partial C}{\partial K}(x, t, T, K)+\frac{1}{2} K^{2} \sigma_{T}^{2}(K) \frac{\partial^{2} C}{\partial K^{2}}(x, t, T, K) .
$$

We also apply our method to Asian options.

We proceed as follows. In Section 2 we recall some notation on the Malliavin calculus on the Wiener space and on stochastic calculus for jump processes. In Section 3 we review the computation of Theta $t$ via the Black-Scholes PDE in the jump-diffusion case using the Greeks Delta and Gamma, and the computation of Theta $_{T}$ via the Dupire PDE for European options in a continuous market. We note that those representations formulas differ in general from the one obtained in this paper. In Section 4, using the Malliavin calculus, we obtain an expression of Theta $T$ in a jump-diffusion model which, in the time homogeneous case, reads

$$
\text { Theta }=e^{-\tau r} \mathbb{E}\left[\Lambda_{\tau}(u, v, w) \phi\left(S_{\tau}\right)+\int_{-\infty}^{+\infty}\left(\phi\left(S_{\tau}+c\left(S_{\tau}\right) y\right)-\phi\left(S_{\tau}\right)\right) \mu(d y)\right],
$$

with arbitrary payoff function $\phi(\cdot)$ and $S_{0}=x$. Here, $\Lambda_{\tau}(u, v, w)$ is a random weight which is explicitly computed, $\mu(d x)$ and $c(\cdot)$ are respectively the (finite) Lévy measure and the volatility coefficient of the compound Poisson process driving $\left(S_{t}\right)_{t \in \mathbb{R}_{+}}$. As in 
[4], [5], differentiation is performed only with respect to the Brownian component of the price process. The above expression is independent of the functional parameters $u, v, w$, and in Section 5 we determine the parameters yielding the best numerical performance by minimization of the variance of the weight $\Lambda_{\tau}(u, v, w)$, and find that this minimum is attained when $u, v, w$ are constant functions. Asian options are considered in Section 6. Simulations for digital and European options, using the Monte Carlo method, are presented in Section 7 to compare the performance of the finite difference method to that of the Malliavin calculus approach. A localization approach is presented in Section 8 to reduce the singularity points phenomenon on the simulation graphs.

\section{Notation}

In this section we recall some facts and notation on the Malliavin calculus on the Wiener space, cf e.g. [10], and on stochastic calculus with jumps, see e.g. and [3] for a recent introduction with references. We work on a product

$$
(\Omega, P)=\left(\Omega_{W} \times \Omega_{X}, P_{W} \otimes P_{X}\right)
$$

of probability spaces on which are respectively defined a standard Brownian motion $\left(W_{t}\right)_{t \in \mathbb{R}_{+}}$and a compound Poisson process $\left(X_{t}\right)_{t \in \mathbb{R}_{+}}$independent of $\left(W_{t}\right)_{t \in \mathbb{R}_{+}}$, with Lévy measure $\mu(d y)$ and finite intensity

$$
\lambda=\int_{-\infty}^{\infty} y \mu(d y)
$$

which can be represented as

$$
X_{t}=\sum_{k=1}^{N_{t}} U_{k}, \quad t \in \mathbb{R}_{+},
$$

where $\left(N_{t}\right)_{t \in \mathbb{R}_{+}}$is a Poisson process with intensity $\lambda$ and $\left(U_{k}\right)_{k \geq 1}$ is an i.i.d. sequence of random variables with probability distribution $\nu(d x)=\lambda^{-1} \mu(d x)$. We denote by $\left(\mathcal{F}_{t}\right)_{t \in \mathbb{R}_{+}}$the filtration generated by $\left(W_{t}, X_{t}\right)_{t \in \mathbb{R}_{+}}$.

We consider gradient and divergence operators $D$ and $\delta$ acting on the continuous component of jump-diffusion random functionals. Let $D: L^{2}(\Omega) \rightarrow L^{2}\left(\Omega \times \mathbb{R}_{+}\right)$ denote the (unbounded) Malliavin gradient on the Wiener space, i.e.

$$
D_{t} F\left(\omega_{W}, \omega_{X}\right)=\sum_{k=1}^{n} 1_{\left[0, t_{k}\right]}(t) \partial_{k} f\left(W_{t_{1}}, \ldots, W_{t_{n}}, \omega_{X}\right)
$$

$\mathrm{RR} \mathrm{n}^{\circ} 5829$ 
for $F$ a random variable of the form

$$
F\left(\omega_{W}, \omega_{X}\right)=f\left(W_{t_{1}}, \ldots, W_{t_{n}}, \omega_{X}\right),
$$

where $f\left(\cdot, \omega_{X}\right) \in \mathcal{C}_{b}^{\infty}\left(\mathbb{R}^{n}\right), P_{X}\left(d \omega_{X}\right)$-a.s., is uniformly bounded on $\Omega_{X}$. Denote by $\langle\cdot, \cdot\rangle_{L^{2}\left(\mathbb{R}_{+}\right)}$and $\|\cdot\|$ the scalar product and norm in $L^{2}\left(\mathbb{R}_{+}\right)$, and define

$$
D_{u} F=\langle u, D F\rangle, \quad u \in L^{2}\left(\Omega \times \mathbb{R}_{+}\right) .
$$

Let

$$
I_{n}\left(f_{n}\right)\left(\omega_{W}, \omega_{X}\right)=n ! \int_{0}^{\infty} \cdots \int_{0}^{t_{2}} f_{n}\left(t_{1}, \ldots, t_{n}, \omega_{X}\right) d W_{t_{1}} \cdots d W_{t_{n}}
$$

denote the multiple stochastic integral of the symmetric function $f_{n} \in L^{2}\left(\mathbb{R}_{+}^{n} \times \Omega_{X}\right)$ with respect to Brownian motion $\left(W_{t}\right)_{t \in \mathbb{R}_{+}}$. Recall that we have

$$
D_{t} I_{n}\left(f_{n}\right)=n I_{n-1}\left(f_{n}\left(\cdot, t, \omega_{X}\right)\right), \quad t \in \mathbb{R}_{+},
$$

and the isometry formula

$$
\mathbb{E}\left[I_{n}\left(f_{n}\right) I_{m}\left(g_{m}\right)\right]=n ! 1_{\{n=m\}} \mathbb{E}\left[\left\langle f_{n}, g_{m}\right\rangle_{L^{2}\left(\mathbb{R}_{+}^{n}\right)}\right] .
$$

Any $F \in L^{2}\left(\Omega_{W} \times \Omega_{X}\right) \simeq L^{2}\left(\Omega_{W} ; L^{2}\left(\Omega_{X}\right)\right)$ admits a chaos decomposition of the form

$$
F=E[F]+\sum_{n=1}^{\infty} I_{n}\left(f_{n}\right),
$$

where $f_{n} \in L^{2}\left(\mathbb{R}_{+}^{n} \times \Omega_{X}\right), n \geq 1$, and the domain $\operatorname{Dom}(D)$ of $D$ consists in the set of functionals $F$ written as (2.2) and satisfying

$$
\mathbb{E}\left[\sum_{n=1}^{\infty} n ! n\left\|f_{n}\right\|_{L^{2}\left(\mathbb{R}_{+}^{n}\right)}^{2}\right]<\infty .
$$

In the sequel we will generally drop the indices $\omega_{W}, \omega_{X}$. The (unbounded) divergence operator $\delta: L^{2}\left(\Omega \times \mathbb{R}_{+}\right) \rightarrow L^{2}(\Omega)$ adjoint of $D$, also called the Skorohod integral, satisfies the duality relation

$$
\mathbb{E}[\langle D F, u\rangle]=\mathbb{E}[F \delta(u)], \quad F \in \operatorname{Dom}(D), \quad u \in \operatorname{Dom}(\delta),
$$

and the divergence formula

$$
\delta(u F)=F \delta(u)-D_{u} F, \quad u \in \operatorname{Dom}(\delta), \quad F \in \operatorname{Dom}(D),
$$


which shows that $u F \in \operatorname{Dom}(\delta)$ provided $u F \in L^{2}\left(\Omega \times \mathbb{R}_{+}\right)$and the right-hand side belongs to $L^{2}(\Omega)$. Recall also that $\delta$ coincides with Itô's stochastic integral on square-integrable adapted processes, in particular

$$
\delta(u)=\int_{0}^{\infty} u_{t} d W_{t}
$$

for all adapted and square-integrable process $\left(u_{t}\right)_{t \in \mathbb{R}_{+}}$, and

$$
\delta(u)=I_{1}(u), \quad u \in L^{2}\left(\mathbb{R}_{+}\right) .
$$

In the sequel we will consider a Markovian jump-diffusion price process $\left(S_{t}\right)_{t \in \mathbb{R}_{+}}$ given by

$$
\left\{\begin{array}{l}
d S_{t}=a_{t}\left(S_{t}\right) d t+b_{t}\left(S_{t}\right) d W_{t}+c_{t}\left(S_{t^{-}}\right) d X_{t} \\
S_{0}=x
\end{array}\right.
$$

where $a_{t}(\cdot), b_{t}(\cdot), c_{t}(\cdot)$ are $\mathcal{C}^{1}$ Lipschitz functions, uniformly in $t \in[0, T], T>0$. In the case of a time homogeneous geometric model under the risk neutral measure, the coefficients $a(\cdot), b(\cdot)$ and $c(\cdot)$ will be given by

$$
\left\{\begin{array}{l}
a(y)=y(r-\lambda \eta(y)), \\
b(y)=y \sigma(y), \\
c(y)=y \eta(y),
\end{array}\right.
$$

where $r$ and $\sigma(\cdot), \eta(\cdot)$ represent the interest rate, and the continuous and jump volatility functions.

Itô's formula for $\left(S_{t}\right)_{t \in \mathbb{R}_{+}}$reads

$$
\begin{aligned}
\phi\left(S_{t}\right)= & \phi\left(S_{s}\right)+\int_{s}^{t} \phi^{\prime}\left(S_{u}\right) a_{u}\left(S_{u}\right) d u+\int_{s}^{t} \phi^{\prime}\left(S_{u}\right) b_{u}\left(S_{u}\right) d W_{u} \\
& +\frac{1}{2} \int_{s}^{t} \phi^{\prime \prime}\left(S_{u}\right) b_{u}^{2}\left(S_{u}\right) d u+\sum_{s<u \leq t}\left(\phi\left(S_{u^{-}}+c_{u}\left(S_{u^{-}}\right) \Delta X_{u}\right)-\phi\left(S_{u^{-}}\right)\right),
\end{aligned}
$$

$0 \leq s \leq t$, and yields

$$
\mathbb{E}\left[\phi\left(S_{t}\right)\right]=\mathbb{E}\left[\phi\left(S_{s}\right)\right]+\mathbb{E}\left[\int_{s}^{t} \phi^{\prime}\left(S_{u}\right) a_{u}\left(S_{u}\right) d u\right]+\frac{1}{2} \mathbb{E}\left[\int_{s}^{t} \phi^{\prime \prime}\left(S_{u}\right) b_{u}^{2}\left(S_{u}\right) d u\right]
$$

$\mathrm{RR} \mathrm{n}^{\circ} 5829$ 


$$
+\lambda \mathbb{E}\left[\int_{s}^{t} \int_{-\infty}^{+\infty}\left(\phi\left(S_{u}+z c_{u}\left(S_{u}\right)\right)-\phi\left(S_{u}\right)\right) \nu(d z) d u\right] .
$$

For example, if

$$
X_{t}=a_{1} N_{t}^{1}+\cdots+a_{d} N_{t}^{d}, \quad t \in \mathbb{R}_{+},
$$

where $\left(N_{t}^{k}\right)_{t \in \mathbb{R}_{+}}, k=1, \ldots, d$, are independent Poisson processes with respective intensities $\lambda_{1}, \ldots, \lambda_{d}$, we have $\lambda=\lambda_{1}+\cdots+\lambda_{d}$ and

$$
\nu(d x)=\frac{\lambda_{1}}{\lambda} \delta_{a_{1}}(d x)+\cdots+\frac{\lambda_{d}}{\lambda} \delta_{a_{d}}(d x),
$$

and

$$
\begin{aligned}
\mathbb{E}\left[\phi\left(S_{t}\right)\right]= & \mathbb{E}\left[\phi\left(S_{s}\right)\right]+\mathbb{E}\left[\int_{s}^{t} \phi^{\prime}\left(S_{u}\right) a_{u}\left(S_{u}\right) d u\right]+\frac{1}{2} \mathbb{E}\left[\int_{s}^{t} \phi^{\prime \prime}\left(S_{u}\right) b_{u}^{2}\left(S_{u}\right) d u\right] \\
& +\sum_{k=1}^{d} \lambda_{k} \mathbb{E}\left[\int_{s}^{t}\left(\phi\left(S_{u}+a_{k} c_{u}\left(S_{u}\right)\right)-\phi\left(S_{u}\right)\right) d u\right], \quad 0 \leq s \leq t .
\end{aligned}
$$

In the linear case we let

$$
\left\{\begin{array}{l}
a(y)=(r-\lambda \eta) y \\
b(y)=\sigma y \\
c(y)=\eta y
\end{array}\right.
$$

and get

$$
\left\{\begin{array}{l}
d S_{s}=r S_{s} d s+\sigma S_{s} d W_{s}+\eta S_{s^{-}}\left(d X_{s}-\lambda d s\right) \\
S_{0}=x
\end{array}\right.
$$

with solution

$$
S_{t}=x \exp \left(\left(r-\lambda \eta-\frac{\sigma^{2}}{2}\right) t+\sigma W_{t}\right) \prod_{0<s \leq t}\left(1+\eta \Delta X_{s}\right), \quad t \in \mathbb{R}_{+} .
$$

If $\left(X_{t}\right)_{t \in \mathbb{R}_{+}}$has the form (2.5) we obtain

$$
S_{t}=x \exp \left(\left(r-\lambda \eta-\frac{\sigma^{2}}{2}\right) t+\sigma W_{t}\right)\left(1+\eta a_{1}\right)^{N_{t}^{1}} \cdots\left(1+\eta a_{d}\right)^{N_{t}^{d}}, \quad t \in \mathbb{R}_{+}
$$




\section{PDE approaches to Theta}

In this section we review the computation of Theta $_{t}$ and Theta $T$ in particular cases, using respectively the Black-Scholes and Dupire PDEs.

Consider an option with payoff function $\phi$ and price

$$
C(x, t, T)=e^{-\int_{t}^{T} r_{s} d s} \mathbb{E}\left[\phi\left(S_{T}\right) \mid S_{t}=x\right] .
$$

Since $t \mapsto e^{\int_{t}^{T} r_{s} d s} C\left(S_{t}, t, T\right)$ is a martingale, from (2.4), $C(x, t, T)$ satisfies the BlackScholes PDE

$$
\begin{aligned}
\text { Theta }_{t}= & \frac{\partial C}{\partial t}(x, t, T) \\
= & r_{t} C(x, t, T)-a_{t}(x) \frac{\partial C}{\partial x}(x, t, T)-\frac{1}{2} b_{t}^{2}(x) \frac{\partial^{2} C}{\partial x^{2}}(x, t, T) \\
& \left.-\lambda \int_{-\infty}^{+\infty}\left(C\left(x+z c_{t}(x), t, T\right)\right)-C(x, t, T)\right) \nu(d z),
\end{aligned}
$$

with

$$
\text { Delta }=\frac{\partial C}{\partial x}(x, t, T)=e^{-\int_{t}^{T} r_{s} d s} \mathbb{E}\left[Y_{T} \phi^{\prime}\left(S_{T}\right) \mid S_{t}=x\right],
$$

where $\left(Y_{t}\right)_{t \in \mathbb{R}_{+}}=\left(\partial_{x} S_{t}\right)_{t \in \mathbb{R}_{+}}$is the first variation process solution of

$$
\left\{\begin{array}{l}
d Y_{t}=a_{t}^{\prime}\left(S_{t}\right) Y_{t} d t+b_{t}^{\prime}\left(S_{t}\right) Y_{t} d W_{t}+c_{t}^{\prime}\left(S_{t^{-}}\right) Y_{t^{-}} d X_{t} \\
Y_{0}=1
\end{array}\right.
$$

and

$$
\text { Gamma }=\frac{\partial^{2} C}{\partial x^{2}}(x, t, T)=e^{-\int_{t}^{T} r_{s} d s} \mathbb{E}\left[Z_{T} \phi^{\prime}\left(S_{T}\right)+\left(Y_{T}\right)^{2} \phi^{\prime \prime}\left(S_{T}\right) \mid S_{t}=x\right],
$$

where $\left(Z_{t}\right)_{t \in \mathbb{R}_{+}}=\left(\partial_{x}^{2} S_{t}\right)_{t \in \mathbb{R}_{+}}$is the second variation process. Here, $a_{t}^{\prime}(z), b_{t}^{\prime}(z)$ and $c_{t}^{\prime}(z)$ denote the partial derivatives of these functions with respect to $z$.

The first and second derivatives on $\phi$ in the expressions (3.2), (3.3) of Delta and Gamma can be removed via integration by parts and the Malliavin calculus, as in e.g. [5], [4], to yield an expression for Theta . However the computation of Gamma 
directly involves the first and second variation processes $Y_{t}$ and $Z_{t}$. Namely in [7], the expression (3.2) is rewritten as

$$
\text { Delta }=\frac{\partial C}{\partial x}(x, t, T)=\frac{e^{-\int_{t}^{T} r_{s} d s}}{T-t} \mathbb{E}\left[\phi\left(S_{T}\right) \int_{t}^{T} \frac{Y_{s}}{b_{s}\left(S_{s}\right)} d W_{s} \mid S_{t}=x\right],
$$

using the expression of the Malliavin derivative of $S_{T} \in \operatorname{Dom}(D)$ in terms of the first variation process as:

$$
\frac{Y_{s}}{b_{s}\left(S_{s}\right)} D_{s} \phi\left(S_{T}\right)=Y_{T} \phi^{\prime}\left(S_{T}\right), \quad 0 \leq s \leq \tau, \text { a.s. }
$$

cf. [10]. In the next section we present a computation of Theta $T$ by integration by parts which, in the time homogeneous case, yields a different representation for Theta $_{t}=-$ Theta $_{T}$. It does not directly use the first and second variation processes, and involves only elementary Wiener integrals of deterministic functions instead of Itô stochastic integrals of adapted processes as in (3.5).

On the other hand, in the case of European options in a continuous market, i.e. with $c_{t}(\cdot)=0, a_{t}(y)=\alpha_{t} y$, with payoff function $\phi(x)=(x-K)^{+}$, and price

$$
C(x, t, T, K)=e^{-\int_{t}^{T} r_{s} d s} \mathbb{E}\left[\left(S_{T}-K\right)^{+} \mid S_{t}=x\right],
$$

Dupire's formula [6] reads

$$
b_{T}(K)=\sqrt{2 \frac{\left(r_{T}-\alpha_{T}\right) C+\frac{\partial C}{\partial T}+K r_{T} \frac{\partial C}{\partial K}}{K^{2} \frac{\partial^{2} C}{\partial K^{2}}}}
$$

where $\frac{\partial C}{\partial T}(x, t, T, K)$ coincides with Theta $_{T}$. In other terms,

$$
\begin{aligned}
& \operatorname{Theta}_{T}=\frac{\partial C}{\partial T}(x, t, T, K) \\
& =\left(\alpha_{T}-r_{T}\right) C(x, t, T, K)+\frac{K^{2} b_{T}^{2}(K)}{2} \frac{\partial^{2} C}{\partial K^{2}}(x, t, T, K)-K \alpha_{T} \frac{\partial C}{\partial K}(x, t, T, K),
\end{aligned}
$$

where $-\frac{\partial^{2} C}{\partial y^{2}}(x, t, T, y)$ coincides with the density function $d P\left(S_{T}=y \mid S_{t}=x\right) / d y$. Relation (3.6) can be proved by application of $(2.4)$ on $[0, T]$, differentiation with 
respect to $T$, and integration by parts with respect to $d y$ on $\mathbb{R}$. The computation of Theta $_{T}$ presented in the next section follows the same steps, replacing integration by parts on $\mathbb{R}$ with the duality formula on the Wiener space. As such it can be viewed as a generalization of Dupire's argument to arbitrary payoff functions in a jump diffusion market.

\section{Computation of Theta}

Consider an option with payoff function $\phi$ and price

$$
C(x, t, T)=e^{-\int_{t}^{T} r_{s} d s} \mathbb{E}\left[\phi\left(S_{T}\right) \mid S_{t}=x\right] .
$$

Theta $_{T}$ can be approximated by finite differences as

$$
\operatorname{Theta}_{T}=\frac{C(x, t,(1+\varepsilon) T)-C(x, t,(1-\varepsilon) T)}{2 T \varepsilon} .
$$

Alternatively, the derivative with respect to $T$ can be put inside the expectation if $\phi$ is differentiable.

Consider $\left(S_{t, s}^{x}\right)_{s \in[t, \infty)}$ given by the jump-diffusion equation

$$
\left\{\begin{array}{l}
d S_{t, s}^{x}=a_{s}\left(S_{t, s}^{x}\right) d s+b_{s}\left(S_{t, s}^{x}\right) d W_{s}+c_{s}\left(S_{t, s^{-}}^{x}\right) d X_{s} \\
S_{t, t}^{x}=x
\end{array}\right.
$$

Using Itô's formula and (2.4) we have:

$$
\begin{aligned}
C(x, t, T) & =e^{-\int_{t}^{T} r_{s} d s} \mathbb{E}\left[\phi\left(S_{t, T}^{x}\right)\right] \\
= & \phi(x)-\mathbb{E}\left[\int_{t}^{T} r_{s} e^{-\int_{t}^{s} r_{p} d p} \phi\left(S_{t, s}^{x}\right) d s\right] \\
+ & \mathbb{E}\left[\int_{t}^{T} e^{-\int_{t}^{s} r_{p} d p} \phi^{\prime}\left(S_{t, s}^{x}\right) a_{s}\left(S_{t, s}^{x}\right) d s\right]+\mathbb{E}\left[\int_{t}^{T} e^{-\int_{t}^{s} r_{p} d p} \phi^{\prime}\left(S_{t, s}^{x}\right) b_{s}\left(S_{t, s}^{x}\right) d W_{s}\right] \\
+ & \frac{1}{2} \mathbb{E}\left[\int_{t}^{T} e^{-\int_{t}^{s} r_{p} d p} \phi^{\prime \prime}\left(S_{t, s}^{x}\right) b_{s}^{2}\left(S_{t, s}^{x}\right) d s\right] \\
+ & \lambda \mathbb{E}\left[\int_{t}^{T} e^{-\int_{t}^{s} r_{p} d p} \int_{-\infty}^{+\infty}\left(\phi\left(S_{t, s}^{x}+z c_{s}\left(S_{t, s}^{x}\right)\right)-\phi\left(S_{t, s}^{x}\right)\right) \nu(d z) d s\right]
\end{aligned}
$$

$\operatorname{RR} \mathrm{n}^{\circ} 5829$ 


$$
\begin{aligned}
= & \phi(x)-\int_{t}^{T} r_{s} e^{-\int_{t}^{s} r_{p} d p_{1}} \mathbb{E}\left[\phi\left(S_{t, s}^{x}\right)\right] d s+\int_{t}^{T} e^{-\int_{t}^{s} r_{p} d p} \mathbb{E}\left[\phi^{\prime}\left(S_{t, s}^{x}\right) a_{s}\left(S_{t, s}^{x}\right)\right] d s \\
& +\frac{1}{2} \int_{t}^{T} e^{-\int_{t}^{s} r_{p} d p} \mathbb{E}\left[\phi^{\prime \prime}\left(S_{t, s}^{x}\right) b_{s}^{2}\left(S_{t, s}^{x}\right)\right] d s \\
& +\lambda \int_{t}^{T} e^{-\int_{t}^{s} r_{p} d p_{1}} \mathbb{E}\left[\int_{-\infty}^{+\infty}\left(\phi\left(S_{t, s}^{x}+z c_{s}\left(S_{t, s}^{x}\right)\right)-\phi\left(S_{t, s}^{x}\right)\right) \nu(d z)\right] d s,
\end{aligned}
$$

hence $\operatorname{Theta}_{T}$ can be expressed as

$$
\begin{aligned}
\operatorname{Theta}_{T}= & \frac{\partial}{\partial T}\left(e^{-\int_{t}^{T} r_{s} d s} \mathbb{E}\left[\phi\left(S_{t, T}^{x}\right)\right]\right) \\
= & -r_{T} e^{-\int_{t}^{T} r_{p} d p} \mathbb{E}\left[\phi\left(S_{t, T}^{x}\right)\right]+e^{-\int_{t}^{T} r_{p} d p} \mathbb{E}\left[\phi^{\prime}\left(S_{t, T}^{x}\right) a_{T}\left(S_{t, T}^{x}\right)\right] \\
& +\frac{1}{2} e^{-\int_{t}^{T} r_{p} d p} \mathbb{E}\left[\phi^{\prime \prime}\left(S_{t, T}^{x}\right) b_{T}^{2}\left(S_{t, T}^{x}\right)\right] \\
& +\lambda e^{-\int_{t}^{T} r_{s} d s} \mathbb{E}\left[\int_{-\infty}^{+\infty}\left(\phi\left(S_{t, T}^{x}+z c_{T}\left(S_{t, T}^{x}\right)\right)-\phi\left(S_{t, T}^{x}\right)\right) \nu(d z)\right]
\end{aligned}
$$

which differs from (3.1), including in the time-homogeneous case. Note that unlike the PDE approach of Section 3, this method does not seem to be applicable to the computation of Theta $t$ in a time-inhomogeneous setting. The above expression fails when $\phi$ is not twice differentiable. The aim of Proposition 4.1 below is to present a Malliavin type formula for Theta $T$, which avoids the use of finite differences and does not require any smoothness on $\phi$. This will allow us in particular to consider non-smooth payoff functions, as e.g. in the case of digital options. The derivatives on $\phi$ will be removed by integration by parts on the Wiener space, using the expression

$$
\phi^{\prime}\left(S_{t, T}^{x}\right)=\frac{D_{u} \phi\left(S_{t, T}^{x}\right)}{D_{u} S_{t, T}^{x}}, \quad u \in L^{2}([t, T]) .
$$

The jump component of the above formula is left untouched since it does not contain any derivative.

Given $u, v, w \in L^{2}([t, T])$ such that $D_{u} S_{t, T}^{x}, D_{v} S_{t, T}^{x}, D_{w} S_{t, T}^{x}$ are a.s. non-zero, let the weight $\Lambda_{t, T}(u, v, w)$ be defined by

$\Lambda_{t, T}(u, v, w)=-a_{T}^{\prime}\left(S_{t, T}^{x}\right)-r_{T}+a_{T}\left(S_{t, T}^{x}\right)\left(\frac{I_{1}(u)}{D_{u} S_{t, T}^{x}}+\frac{D_{u}^{2} S_{t, T}^{x}}{\left|D_{u} S_{t, T}^{x}\right|^{2}}\right)$ 


$$
\begin{aligned}
& +\frac{1}{2}\left(\left(\frac{b_{T}^{2}\left(S_{t, T}^{x}\right)}{D_{v} S_{t, T}^{x}} I_{1}(v)-2 b_{T}\left(S_{t, T}^{x}\right) b_{T}^{\prime}\left(S_{t, T}^{x}\right)+\frac{b_{T}^{2}\left(S_{t, T}^{x}\right) D_{v}^{2} S_{t, T}^{x}}{\left|D_{v} S_{t, T}^{x}\right|^{2}}\right)\left(\frac{I_{1}(w)}{D_{w} S_{t, T}^{x}}+\frac{D_{w}^{2} S_{t, T}^{x}}{\left|D_{w} S_{t, T}^{x}\right|^{2}}\right)\right. \\
& +\frac{b_{T}^{2}\left(S_{t, T}^{x}\right)}{D_{w} S_{t, T}^{x} D_{v} S_{t, T}^{x}}\left(I_{1}(v) \frac{D_{w} D_{v} S_{t, T}^{x}}{D_{v} S_{t, T}^{x}}-\langle v, w\rangle-\frac{D_{w} D_{v}^{2} S_{t, T}^{x}}{D_{v} S_{t, T}^{x}}+2 \frac{D_{w} D_{v} S_{t, T}^{x} D_{v}^{2} S_{t, T}^{x}}{\left|D_{v} S_{t, T}^{x}\right|^{2}}\right) \\
& \left.-\frac{2 b_{T}^{\prime}\left(S_{t, T}^{x}\right) b_{T}\left(S_{t, T}^{x}\right)}{D_{v} S_{t, T}^{x}}\left(I_{1}(v)+\frac{D_{v}^{2} S_{t, T}^{x}}{D_{v} S_{t, T}^{x}}\right)\right)+b_{T}^{\prime}\left(S_{t, T}^{x}\right)^{2}+b_{T}^{\prime \prime}\left(S_{t, T}^{x}\right) b_{T}\left(S_{t, T}^{x}\right) .
\end{aligned}
$$

Recall that the Wiener integrals $I_{1}(u), I_{1}(v)$ and $I_{1}(w)$ in the above formula are centered Gaussian random variables.

Proposition 4.1 Let $u, v, w \in L^{2}([t, T])$ such that $D_{u} S_{t, T}^{x}, D_{v} S_{t, T}^{x}, D_{w} S_{t, T}^{x}$ are a.s. non-zero, assume that $\Lambda_{t, T}(u, v, w) \in L^{2}(\Omega)$, and let $\phi: \mathbb{R} \rightarrow \mathbb{R}$ be a Lipschitz function. We have

Theta $_{T}=$

$$
e^{-\int_{t}^{T} r_{s} d s} \mathbb{E}\left[\Lambda_{t, T}(u, v, w) \phi\left(S_{t, T}^{x}\right)+\lambda \int_{-\infty}^{+\infty}\left(\phi\left(S_{t, T}^{x}+z c_{T}\left(S_{t, T}^{x}\right)\right)-\phi\left(S_{t, T}^{x}\right)\right) \nu(d z)\right] .
$$

Proof. Using (2.3) and (4.4) we get for $u \in L^{2}([t, T])$ and $\phi, g_{T}$, sufficiently smooth:

$$
\begin{aligned}
\mathbb{E} & {\left[\phi^{\prime}\left(S_{t, T}^{x}\right) g_{T}\left(S_{t, T}^{x}\right)\right]=\mathbb{E}\left[\frac{g_{T}\left(S_{t, T}^{x}\right)}{D_{u} S_{t, T}^{x}} D_{u} \phi\left(S_{t, T}^{x}\right)\right] } \\
= & \mathbb{E}\left[\left\langle D \phi\left(S_{t, T}^{x}\right), \frac{g_{T}\left(S_{t, T}^{x}\right)}{D_{u} S_{t, T}^{x}} u\right\rangle\right] \\
= & \mathbb{E}\left[\phi\left(S_{t, T}^{x}\right) \delta\left(\frac{g_{T}\left(S_{t, T}^{x}\right)}{D_{u} S_{t, T}^{x}} u\right)\right] \\
= & \mathbb{E}\left[\phi\left(S_{t, T}^{x}\right)\left(\frac{g_{T}\left(S_{t, T}^{x}\right)}{D_{u} S_{t, T}^{x}} I_{1}(u)-D_{u}\left(\frac{g_{T}\left(S_{t, T}^{x}\right)}{D_{u} S_{t, T}^{x}}\right)\right)\right] \\
= & \mathbb{E}\left[\phi\left(S_{t, T}^{x}\right)\left(\frac{g_{T}\left(S_{t, T}^{x}\right)}{D_{u} S_{t, T}^{x}} I_{1}(u)-g_{T}^{\prime}\left(S_{t, T}^{x}\right)+\frac{g_{T}\left(S_{t, T}^{x}\right) D_{u}^{2} S_{t, T}^{x}}{\left|D_{u} S_{t, T}^{x}\right|^{2}}\right)\right] .
\end{aligned}
$$

With $g_{T}(\cdot)=a_{T}(\cdot)$ we obtain

$\mathbb{E}\left[\phi^{\prime}\left(S_{t, T}^{x}\right) a_{T}\left(S_{t, T}^{x}\right)\right]=\mathbb{E}\left[\phi\left(S_{t, T}^{x}\right)\left(\frac{a_{T}\left(S_{t, T}^{x}\right)}{D_{u} S_{t, T}^{x}} I_{1}(u)-a_{T}^{\prime}\left(S_{t, T}^{x}\right)+\frac{a_{T}\left(S_{t, T}^{x}\right) D_{u}^{2} S_{t, T}^{x}}{\left|D_{u} S_{t, T}^{x}\right|^{2}}\right)\right]$, 
while $g_{T}(\cdot)=b_{T}^{2}(\cdot)$ yields

$$
\mathbb{E}\left[\phi^{\prime \prime}\left(S_{t, T}^{x}\right) b_{T}^{2}\left(S_{t, T}^{x}\right)\right]=\mathbb{E}\left[\phi^{\prime}\left(S_{t, T}^{x}\right) \Gamma_{t, T}(v)\right],
$$

where

$$
\Gamma_{t, T}(v)=\frac{b_{T}^{2}\left(S_{t, T}^{x}\right)}{D_{v} S_{t, T}^{x}} I_{1}(v)-2 b_{T}\left(S_{t, T}^{x}\right) b_{T}^{\prime}\left(S_{t, T}^{x}\right)+\frac{b_{T}^{2}\left(S_{t, T}^{x}\right) D_{v}^{2} S_{t, T}^{x}}{\left|D_{v} S_{t, T}^{x}\right|^{2}} .
$$

By a similar argument we get

$$
\begin{aligned}
& \mathbb{E} {\left[\phi^{\prime \prime}\left(S_{t, s}^{x}\right) b_{s}^{2}\left(S_{t, s}^{x}\right)\right]=\mathbb{E}\left[\phi^{\prime}\left(S_{t, T}^{x}\right) \Gamma_{t, T}(v)\right] } \\
&= \mathbb{E}\left[\Gamma_{t, T}(v) \frac{D_{w} \phi\left(S_{t, T}^{x}\right)}{D_{w}\left(S_{t, T}^{x}\right)}\right] \\
&=\mathbb{E}\left[\phi\left(S_{t, T}^{x}\right) \delta\left(w \frac{\Gamma_{t, T}(v)}{D_{w} S_{t, T}^{x}}\right)\right] \\
&=\mathbb{E}\left[\phi\left(S_{t, T}^{x}\right)\left(\frac{\Gamma_{t, T}(v)}{D_{w} S_{t, T}^{x}} I_{1}(w)-D_{w}\left(\frac{\Gamma_{t, T}(v)}{D_{w} S_{t, T}^{x}}\right)\right)\right] \\
&=\mathbb{E}\left[\phi ( S _ { t , T } ^ { x } ) \left(\frac{\Gamma_{t, T}(v)}{D_{w} S_{t, T}^{x}}\left(I_{1}(w)+\frac{D_{w}^{2} S_{t, T}^{x}}{D_{w} S_{t, T}^{x}}\right)-\frac{2 b_{T}^{\prime}\left(S_{t, T}^{x}\right) b_{T}\left(S_{t, T}^{x}\right)}{D_{v} S_{t, T}^{x}}\left(I_{1}(v)+\frac{D_{v}^{2} S_{t, T}^{x}}{D_{v} S_{t, T}^{x}}\right)\right.\right. \\
&+\frac{b_{T}^{2}\left(S_{t, T}^{x}\right)}{D_{w} S_{t, T}^{x} D_{v} S_{t, T}^{x}}\left(\frac{I_{1}(v) D_{w} D_{v} S_{t, T}^{x}}{D_{v} S_{t, T}^{x}}-\langle v, w\rangle-\frac{D_{w} D_{v}^{2} S_{t, T}^{x}}{D_{v} S_{t, T}^{x}}+\frac{2 D_{w} D_{v} S_{t, T}^{x} D_{v}^{2} S_{t, T}^{x}}{\left|D_{v} S_{t, T}^{x}\right|^{2}}\right) \\
&\left.\left.+2 b_{T}^{\prime}\left(S_{t, T}^{x}\right)^{2}+2 b_{T}^{\prime \prime}\left(S_{t, T}^{x}\right) b_{T}\left(S_{t, T}^{x}\right)\right)\right] .
\end{aligned}
$$

Summing (4.5) with the above relation and using (4.3) we obtain

$$
\begin{aligned}
& \Lambda_{t, T}(u, v, w)=-a_{T}^{\prime}\left(S_{t, T}^{x}\right)-r_{T}+a_{T}\left(S_{t, T}^{x}\right)\left(\frac{I_{1}(u)}{D_{u} S_{t, T}^{x}}+\frac{D_{u}^{2} S_{t, T}^{x}}{\left|D_{u} S_{t, T}^{x}\right|^{2}}\right) \\
& \quad+\frac{1}{2}\left(\Gamma_{t, T}(v)\left(\frac{I_{1}(w)}{D_{w} S_{t, T}^{x}}+\frac{D_{w}^{2} S_{t, T}^{x}}{\left|D_{w} S_{t, T}^{x}\right|^{2}}\right)-\frac{2 b_{T}^{\prime}\left(S_{t, T}^{x}\right) b_{T}\left(S_{t, T}^{x}\right)}{D_{v} S_{t, T}^{x}}\left(I_{1}(v)+\frac{D_{v}^{2} S_{t, T}^{x}}{D_{v} S_{t, T}^{x}}\right)\right. \\
& \left.\quad+\frac{b_{T}^{2}\left(S_{t, T}^{x}\right)}{D_{w} S_{t, T}^{x} D_{v} S_{t, T}^{x}}\left(I_{1}(v) \frac{D_{w} D_{v} S_{t, T}^{x}}{D_{v} S_{t, T}^{x}}-\langle v, w\rangle-\frac{D_{w} D_{v}^{2} S_{t, T}^{x}}{D_{v} S_{t, T}^{x}}+\frac{2 D_{w} D_{v} S_{t, T}^{x} D_{v}^{2} S_{t, T}^{x}}{\left|D_{v} S_{t, T}^{x}\right|^{2}}\right)\right) \\
& \quad+b_{T}^{\prime}\left(S_{t, T}^{x}\right)^{2}+b_{T}^{\prime \prime}\left(S_{t, T}^{x}\right) b_{T}\left(S_{t, T}^{x}\right) .
\end{aligned}
$$

We conclude the proof by approximation of $\phi$ by $\mathcal{C}_{b}^{2}$ functions. 
In the time homogeneous case, denoting $S_{0, \tau}^{x}$ by $S_{\tau}^{x}$ and $\Lambda_{0, \tau}(u, v, w)$ by $\Lambda_{\tau}(u, v, w)$ we have

$$
C(x, t, T)=e^{-(T-t) r} \mathbb{E}\left[\phi\left(S_{T}\right) \mid S_{t}=x\right]=e^{-\tau r} \mathbb{E}\left[\phi\left(S_{\tau}^{x}\right)\right],
$$

with $\tau=T-t$, and

$$
\begin{aligned}
& \text { Theta }_{T}=- \text { Theta }_{t} \\
& \quad=e^{-\tau r} \mathbb{E}\left[\left(\Lambda_{\tau}(u, v, w) \phi\left(S_{\tau}^{x}\right)+\lambda \int_{-\infty}^{+\infty}\left(\phi\left(S_{\tau}^{x}+c\left(S_{\tau}^{x}\right) y\right)-\phi\left(S_{\tau}^{x}\right)\right) \nu(d y)\right)\right],
\end{aligned}
$$

where $u, v, w \in L^{2}([0, \tau])$ are such that $D_{u} S_{\tau}^{x}, D_{v} S_{\tau}^{x}, D_{w} S_{\tau}^{x}$ are a.s. non-zero and $\Lambda_{\tau}(u, v, w) \in L^{2}(\Omega)$. For constant $r, \sigma$ and $\eta$, i.e. in the linear case, we have

$$
\left\{\begin{array}{l}
a(y)=(r-\lambda \eta) y \\
b(y)=\sigma y \\
c(y)=\eta y
\end{array}\right.
$$

and (2.7) yields

$$
D_{u} S_{\tau}^{x}=\sigma \int_{0}^{\tau} u_{s} d s S_{\tau}^{x}
$$

hence $D_{v}^{2} S_{\tau}^{x} /\left|D_{v} S_{\tau}^{x}\right|^{2}=1 / S_{\tau}$ and we get

$$
\Lambda_{\tau}(u, v, w)=-r+\frac{\hat{r}}{\sigma} \frac{I_{1}(u)}{\int_{0}^{\tau} u_{s} d s}-\frac{\sigma}{2} \frac{I_{1}(w)}{\int_{0}^{\tau} w_{s} d s}+\frac{I_{2}(v \circ w)}{2 \int_{0}^{\tau} v_{s} d s \int_{0}^{\tau} w_{s} d s},
$$

where $\hat{r}=r-\lambda \eta$. Theta is then given by

$$
\text { Theta }=e^{-r \tau} \mathbb{E}\left[\Lambda_{\tau}(u, v, w) \phi\left(S_{\tau}^{x}\right)+\lambda \int_{-\infty}^{+\infty}\left(\phi\left(S_{\tau}^{x}(1+\eta y)\right)-\phi\left(S_{\tau}^{x}\right)\right) \nu(d y)\right],
$$

i.e. in the model of $(2.5)$ we have

$$
\text { Theta }=e^{-r \tau} \mathbb{E}\left[\Lambda_{\tau}(u, v, w) \phi\left(S_{\tau}^{x}\right)+\sum_{k=1}^{d} \lambda_{k}\left(\phi\left(S_{\tau}^{x}\left(1+\eta a_{k}\right)\right)-\phi\left(S_{\tau}^{x}\right)\right)\right],
$$

and if $\left(X_{t}\right)_{t \in \mathbb{R}_{+}}$is a standard Poisson process with intensity $\lambda$ and jump size $a$, we get

$$
\text { Theta }=e^{-r \tau} \mathbb{E}\left[\Lambda_{\tau}(u, v, w) \phi\left(S_{\tau}^{x}\right)+\lambda\left(\phi\left((1+a \eta) S_{\tau}^{x}\right)-\phi\left(S_{\tau}^{x}\right)\right)\right]
$$

$\mathrm{RR} \mathrm{n}^{\circ} 5829$ 
If $\left(X_{t}\right)_{t \in \mathbb{R}_{+}}$has infinitely many jumps on bounded time intervals, i.e. if $\mu(\mathbb{R})=\infty$, then $\left(S_{t}\right)_{t \in \mathbb{R}_{+}}$is given by

$$
\left\{\begin{array}{l}
d S_{t}=a_{t}\left(S_{t}\right) d t+b_{t}\left(S_{t}\right) d W_{t}+c_{t}\left(S_{t^{-}}\right) d \tilde{X}_{t}, \\
S_{0}=x
\end{array}\right.
$$

where $\left(\tilde{X}_{t}\right)_{t \in \mathbb{R}_{+}}$is the compensated pure jump martingale associated to $\left(X_{t}\right)_{t \in \mathbb{R}_{+}}$. In this case Itô's formula reads

$$
\begin{aligned}
& \phi\left(S_{t}\right)=\phi\left(S_{s}\right)+\int_{s}^{t} \phi^{\prime}\left(S_{u}\right) a_{u}\left(S_{u}\right) d u+\int_{s}^{t} \phi^{\prime}\left(S_{u}\right) b_{u}\left(S_{u}\right) d W_{u}+\int_{s}^{t} \phi^{\prime}\left(S_{u}\right) b_{u}\left(S_{u}\right) d \tilde{X}_{u} \\
& +\frac{1}{2} \int_{s}^{t} \phi^{\prime \prime}\left(S_{u}\right) b^{2}\left(S_{u}\right) d u+\sum_{s<u \leq t}\left(\phi\left(S_{u^{-}}+c_{u}\left(S_{u^{-}}\right) \Delta X_{u}\right)-\phi\left(S_{u^{-}}\right)-c_{u}\left(S_{u^{-}}\right) \Delta X_{u} \phi^{\prime}\left(S_{u^{-}}\right)\right),
\end{aligned}
$$

$0 \leq s \leq t$, to yield

$$
\begin{aligned}
\mathbb{E}\left[\phi\left(S_{t}\right)\right]= & \mathbb{E}\left[\phi\left(S_{s}\right)\right]+\mathbb{E}\left[\int_{s}^{t} \phi^{\prime}\left(S_{u}\right) a_{u}\left(S_{u}\right) d u\right]+\frac{1}{2} \mathbb{E}\left[\int_{s}^{t} \phi^{\prime \prime}\left(S_{u}\right) b_{u}^{2}\left(S_{u}\right) d u\right] \\
& +\lambda \mathbb{E}\left[\int_{s}^{t} \int_{-\infty}^{+\infty}\left(\phi\left(S_{u}+z c_{u}\left(S_{u}\right)\right)-\phi\left(S_{u}\right)-z c_{u}\left(S_{u}\right) \phi^{\prime}\left(S_{u}\right)\right) \nu(d z) d u\right] .
\end{aligned}
$$

However, in this case the last component in $\phi^{\prime}\left(S_{u}\right)$ can not be isolated and dealt with by integration by parts.

\section{Optimization of convergence}

By the results of the preceding section, the value of Theta in the geometric model (2.6) is given by

$$
\text { Theta }=e^{-r \tau} \mathbb{E}\left[\phi\left(S_{\tau}^{x}\right) \Lambda(u, v, w)+\lambda \int_{-\infty}^{+\infty}\left(\phi\left(S_{\tau}^{x}+\eta S_{\tau}^{x} y\right)-\phi\left(S_{\tau}^{x}\right)\right) \nu(d y)\right] .
$$

For any $u \in L^{2}([0, \tau])$ such that $\int_{0}^{\tau} u_{s} d s \neq 0$, letting

$$
\tilde{u}_{t}=\frac{u_{t}}{\int_{0}^{\tau} u_{s} d s}, \quad t \in[0, \tau],
$$


the weight $\Lambda_{\tau}(u, v, w)$ is expressed as

$$
\Lambda_{\tau}(u, v, w)=-r+\frac{\hat{r}}{\sigma} I_{1}(\tilde{u})-\frac{\sigma}{2} I_{1}(\tilde{w})+\frac{1}{2} I_{2}(\tilde{v} \circ \tilde{w}) .
$$

Our goal is now to find functions $u, v, w$ which minimize $\operatorname{Var}\left[\Lambda_{\tau}(u, v, w)\right]$ in this setting.

Proposition 5.1 The infimum on $\operatorname{Var}\left[\Lambda_{\tau}(u, v, w)\right]$ is attained for any non-zero constant functions $u, v, w$ of the form $u_{s}=c_{1}, v_{s}=c_{2}, w_{s}=c_{3}, s \in[0, \tau]$, and is given by

$$
\inf _{u, v, w} \operatorname{Var}\left[\Lambda_{\tau}(u, v, w)\right]=\operatorname{Var}\left[\Lambda_{\mathrm{opt}}\right]=\frac{1}{2 \tau^{2}}+\frac{1}{\sigma^{2} \tau}\left|\hat{r}-\frac{\sigma^{2}}{2}\right|^{2},
$$

where $\hat{r}=r-\lambda \eta$, with

$$
\Lambda_{\mathrm{opt}}=-r+\frac{W_{\tau}}{\sigma \tau}\left(\hat{r}-\frac{\sigma^{2}}{2}\right)+\frac{1}{2 \tau}\left(\frac{W_{\tau}^{2}}{\tau}-1\right) .
$$

Proof. Recall that the Cauchy-Schwarz inequality yields

$$
\|\tilde{u}\|^{2} \geq \frac{1}{\tau}
$$

with equality if and only if $\tilde{u}_{t}=1 / \tau, t \in[0, \tau]$.

Let

$$
\begin{aligned}
F(u, v, w) & =\operatorname{Var}\left[\Lambda_{\tau}(u, v, w)\right] \\
& =\frac{\hat{r}^{2}}{\sigma^{2}}\|\tilde{u}\|^{2}-\hat{r}\langle\tilde{u}, \tilde{w}\rangle+\frac{\sigma^{2}}{4}\|\tilde{w}\|^{2}+\frac{1}{4}\|\tilde{v}\|^{2}\|\tilde{w}\|^{2}+\frac{1}{4}\langle\tilde{v}, \tilde{w}\rangle^{2} \\
& =\frac{1}{\sigma^{2}}\left\|\hat{r} \tilde{u}-\frac{\sigma^{2}}{2} \tilde{w}\right\|^{2}+\frac{1}{4}\|\tilde{v}\|^{2}\|\tilde{w}\|^{2}+\frac{1}{4}\langle\tilde{v}, \tilde{w}\rangle^{2} .
\end{aligned}
$$

The optimal value of $(u, v, w)$ solves

$$
\left\{\begin{array}{l}
\frac{d}{d \varepsilon} F(u+\varepsilon h, v, w)_{\mid \varepsilon=0}=0 \\
\frac{d}{d \varepsilon} F(u, v+\varepsilon h, w)_{\mid \varepsilon=0}=0 \\
\frac{d}{d \varepsilon} F(u, v, w+\varepsilon h)_{\mid \varepsilon=0}=0
\end{array}\right.
$$

$\operatorname{RR} n^{\circ} 5829$ 
for all $h \in L^{2}([0, \tau])$, i.e.

$$
\begin{aligned}
& \frac{2 \hat{r}^{2}}{\sigma^{2}}\left(\langle h, \tilde{u}\rangle-\|\tilde{u}\|^{2} \int_{0}^{\tau} h_{s} d s\right)-\hat{r}\left(\langle h, \tilde{w}\rangle-\langle\tilde{u}, \tilde{w}\rangle \int_{0}^{\tau} h_{s} d s\right)=0, \\
& \frac{1}{2}\|\tilde{w}\|^{2}\left(\langle h, \tilde{v}\rangle-\|\tilde{v}\|^{2} \int_{0}^{\tau} h_{s} d s\right)+\frac{1}{2}\left(\langle\tilde{v}, \tilde{w}\rangle\langle h, \tilde{w}\rangle-\langle\tilde{v}, \tilde{w}\rangle^{2} \int_{0}^{\tau} h_{s} d s\right)=0,
\end{aligned}
$$

and

$$
\begin{aligned}
& \frac{\sigma^{2}}{2}\left(\langle h, \tilde{w}\rangle-\|\tilde{w}\|^{2} \int_{0}^{\tau} h_{s} d s\right)+\frac{1}{2}\|\tilde{v}\|^{2}\left(\langle h, \tilde{w}\rangle-\|\tilde{w}\|^{2} \int_{0}^{\tau} h_{s} d s\right) \\
& \quad+\frac{1}{2}\left(\langle\tilde{v}, \tilde{w}\rangle\langle h, \tilde{v}\rangle-\langle\tilde{v}, \tilde{w}\rangle^{2} \int_{0}^{\tau} h_{s} d s\right)-\hat{r}\left(\langle h, \tilde{u}\rangle-\langle\tilde{u}, \tilde{w}\rangle \int_{0}^{\tau} h_{s} d s\right)=0 .
\end{aligned}
$$

Clearly, for all $c_{1}, c_{2}, c_{3} \neq 0$ the constant functions $u_{s}=c_{1}, v_{s}=c_{2}, w_{s}=c_{3}$, $s \in[0, \tau]$, are solutions of this problem. Let us show that this solution is unique. For all $h \in L^{2}([0, \tau])$ such that $\int_{0}^{\tau} h_{s} d s=0$, equation (5.4) yields

$$
\left\{\begin{array}{l}
\frac{2 \hat{r}^{2}}{\sigma^{2}}\langle h, \tilde{u}\rangle-\hat{r}\langle h, \tilde{w}\rangle=0 \\
\|\tilde{w}\|^{2}\langle h, \tilde{v}\rangle+\langle\tilde{v}, \tilde{w}\rangle\langle h, \tilde{w}\rangle=0 \\
\sigma^{2}\langle h, \tilde{w}\rangle+\|\tilde{v}\|^{2}\langle h, \tilde{w}\rangle+\langle\tilde{v}, \tilde{w}\rangle\langle h, \tilde{v}\rangle-2 \hat{r}\langle h, \tilde{u}\rangle=0 .
\end{array}\right.
$$

If a solution $(\tilde{u}, \tilde{v}, \tilde{w})$ different from $(1 / \tau, 1 / \tau, 1 / \tau)$ exists, then one can find $h \in$ $L^{2}([0, \tau])$ such that $\int_{0}^{\tau} h_{s} d s=0$ and $(\langle h, \tilde{u}\rangle,\langle h, \tilde{v}\rangle,\langle h, \tilde{w}\rangle) \neq(0,0,0)$, hence the determinant

$$
\|\tilde{v}\|^{2}\|\tilde{w}\|^{2}-|\langle\tilde{v}, \tilde{w}\rangle|^{2}=0
$$

of the above linear system vanishes. From (5.3) and (5.5) we get

$$
\begin{aligned}
F(u, v, w) & =\frac{1}{\sigma^{2}}\left\|\hat{r} \tilde{u}-\frac{\sigma^{2}}{2} \tilde{w}\right\|^{2}+\frac{1}{4}\|\tilde{v}\|^{2}\|\tilde{w}\|^{2}+\frac{1}{4}|\langle\tilde{v}, \tilde{w}\rangle|^{2} \\
& =\frac{1}{\sigma^{2}}\left\|\hat{r} \tilde{u}-\frac{\sigma^{2}}{2} \tilde{w}\right\|^{2}+\frac{1}{2}\|\tilde{v}\|^{2}\|\tilde{w}\|^{2} \\
& \geq \frac{1}{\tau \sigma^{2}}\left|\int_{0}^{\tau}\left(\hat{r} \tilde{u}_{s}-\frac{\sigma^{2}}{2} \tilde{w}_{s}\right) d s\right|^{2}+\frac{1}{2 \tau^{2}}
\end{aligned}
$$




$$
=\frac{1}{\tau \sigma^{2}}\left|\hat{r}-\frac{\sigma^{2}}{2}\right|^{2}+\frac{1}{2 \tau^{2}},
$$

which is greater than the optimal value found when $\tilde{u}, \tilde{v}, \tilde{w}$ are constant functions. Moreover, equality occurs only when $\|\tilde{v}\|^{2}=1 / \tau,\|\tilde{w}\|^{2}=1 / \tau$, and

$$
\left\|\hat{r} \tilde{u}-\frac{\sigma^{2}}{2} \tilde{w}\right\|^{2}=\frac{1}{\tau}\left|\hat{r}-\frac{\sigma^{2}}{2}\right|^{2},
$$

i.e. when $\hat{r} \tilde{u}-\frac{\sigma^{2}}{2} \tilde{w}, \tilde{v}, \tilde{w}$ are constant, which implies that $\tilde{u}$ is also constant, except when $\hat{r}=0$, in which case no constraint is imposed on $u$.

We now need to prove that this solution corresponds to the global minimum of $F$. Since $F(u, v, w) \geq 0$, the infimum exists and we denote it by $l$. By continuity of $F$ on $L^{2}([0, \tau])^{3}$ there exist a sequence $\left(u_{n}, v_{n}, w_{n}\right)_{n \in \mathbb{N}}$ such that

$$
l=\lim _{n \rightarrow \infty} \mathbb{E}\left[\Lambda_{\tau}\left(u_{n}, v_{n}, w_{n}\right)^{2}\right] .
$$

We can assume that $\left(u_{n}, v_{n}, w_{n}\right)$ is bounded: if not, replace it by the bounded sequence

$$
\left(\frac{u_{n}}{\left\|u_{n}\right\|}, \frac{v_{n}}{\left\|v_{n}\right\|}, \frac{w_{n}}{\left\|w_{n}\right\|}\right)_{n \in \mathbb{N}},
$$

on which $F$ takes the same values as on $\left(u_{n}, v_{n}, w_{n}\right)_{n \in \mathbb{N}}$. Under this hypothesis, there exists a sub-sequence $\left(u_{n_{k}}, v_{n_{k}}, w_{n_{k}}\right)_{k \in \mathbb{N}}$ converging weakly to $(u, v, w)$ in $L^{2}([0, \tau])^{3}$. We have

$$
\begin{aligned}
\mathbb{E}\left[\Lambda_{\tau}(u, v, w) \Lambda_{\tau}\left(u_{n_{k}}, v_{n_{k}}, w_{n_{k}}\right)\right] & \\
= & \hat{r}^{2}+\frac{\hat{r}^{2}}{\sigma^{2}}\left\langle\tilde{u}, \tilde{u}_{n_{k}}\right\rangle-\frac{\hat{r}}{2}\left\langle\tilde{u}, \tilde{w}_{n_{k}}\right\rangle-\frac{\hat{r}}{2}\left\langle\tilde{u}_{n_{k}}, \tilde{w}\right\rangle+\frac{\sigma^{2}}{4}\left\langle\tilde{w}, \tilde{w}_{n_{k}}\right\rangle \\
& +\frac{1}{4}\left\langle\tilde{u}, \tilde{u}_{n_{k}}\right\rangle\left\langle\tilde{w}, \tilde{w}_{n_{k}}\right\rangle+\frac{1}{4}\left\langle\tilde{u}, \tilde{w}_{n_{k}}\right\rangle\left\langle\tilde{w}, \tilde{u}_{n_{k}}\right\rangle,
\end{aligned}
$$

and by weak convergence of $\left(u_{n_{k}}, v_{n_{k}}, w_{n_{k}}\right)_{k \in \mathbb{N}}$ to $(u, v, w)$ we get

$$
\lim _{n \rightarrow \infty} \mathbb{E}\left[\Lambda_{\tau}(u, v, w) \Lambda_{\tau}\left(u_{n_{k}}, v_{n_{k}}, w_{n_{k}}\right)\right]=\mathbb{E}\left[\left|\Lambda_{\tau}(u, v, w)\right|^{2}\right]
$$

Moreover,

$$
0 \geq l-\mathbb{E}\left[\left|\Lambda_{\tau}(u, v, w)\right|^{2}\right]
$$

$\mathrm{RR} \mathrm{n}^{\circ} 5829$ 


$$
\begin{aligned}
= & \lim _{n \rightarrow \infty} \mathbb{E}\left[\Lambda_{\tau}\left(u_{n_{k}}, v_{n_{k}}, w_{n_{k}}\right)^{2}\right]-\mathbb{E}\left[\left|\Lambda_{\tau}(u, v, w)\right|^{2}\right] \\
\geq & \lim _{n \rightarrow \infty} \mathbb{E}\left[\left|\Lambda_{\tau}(u, v, w)-\Lambda_{\tau}\left(u_{n_{k}}, v_{n_{k}}, w_{n_{k}}\right)\right|^{2}\right] \\
& +2 \mathbb{E}\left[\Lambda_{\tau}(u, v, w) \Lambda_{\tau}\left(u_{n_{k}}, v_{n_{k}}, w_{n_{k}}\right)\right]-2 \mathbb{E}\left[\left|\Lambda_{\tau}(u, v, w)\right|^{2}\right] \\
\geq & \lim _{n \rightarrow \infty} \mathbb{E}\left[\left(\Lambda_{\tau}(u, v, w)-\Lambda_{\tau}\left(u_{n_{k}}, v_{n_{k}}, w_{n_{k}}\right)\right)^{2}\right] \\
& +2 \lim _{n \rightarrow \infty} \mathbb{E}\left[\Lambda_{\tau}(u, v, w) \Lambda_{\tau}\left(u_{n_{k}}, v_{n_{k}}, w_{n_{k}}\right)\right] \\
& -2 \mathbb{E}\left[\left|\Lambda_{\tau}(u, v, w)\right|^{2}\right] \\
\geq & \lim _{n \rightarrow \infty} \mathbb{E}\left[\left(\Lambda_{\tau}(u, v, w)-\Lambda_{\tau}\left(u_{n_{k}}, v_{n_{k}}, w_{n_{k}}\right)\right)^{2}\right] \\
\geq & 0,
\end{aligned}
$$

hence $\lim _{n \rightarrow \infty} \Lambda_{\tau}\left(u_{n_{k}}, v_{n_{k}}, w_{n_{k}}\right)=\Lambda_{\tau}(u, v, w)$ in $L^{2}(\Omega)$ and

$$
l=\mathbb{E}\left[\left|\Lambda_{\tau}(u, v, w)\right|^{2}\right] .
$$

Thus the global minimum is attained for $\tilde{u}=\tilde{v}=\tilde{w}=1 / \tau$.

Note that $\inf _{u, v, w \in L^{2}([0, \tau])} \operatorname{Var}\left[\Lambda_{\tau}(u, v, w)\right]$ is minimal in terms of $\sigma$ and $r$ when $\left(S_{t}^{x}\right)_{t \in \mathbb{R}_{+}}$is an exponential Brownian motion, i.e. $\hat{r}=\sigma^{2} / 2$. In this case we have

$$
\inf _{u, v, w \in L^{2}([0, \tau])} \operatorname{Var}\left[\Lambda_{\tau}(u, v, w)\right]=\frac{1}{2 \tau^{2}} .
$$

\section{Asian options}

Consider the price process $\left(S_{t}\right)_{t \in \mathbb{R}_{+}}$given by

$$
\left\{\begin{array}{l}
d S_{t}^{x}=a\left(S_{t}^{x}\right) d t+b\left(S_{t}^{x}\right) d W_{t}+\eta S_{t^{-}}^{x} d X_{t}, \\
S_{0}^{x}=x
\end{array}\right.
$$

i.e. we let $c_{t}(z)=\eta z$. Recall that, cf. [11], the price

$$
C(x, y, t, T)=e^{-(T-t) r} \mathbb{E}\left[\left(\frac{1}{T} \int_{0}^{T} S_{u}^{x} d u-K\right)^{+} \mid \mathcal{F}_{t}\right]
$$

at time $t$ of an Asian option with price process $\left(S_{t}^{x}\right)_{t \in \mathbb{R}_{+}}$can be expressed as

$$
C(x, y, t, T)=x e^{-(T-t) r} \mathbb{E}\left[\left(y+\frac{1}{T} \int_{t}^{T} S_{s-t}^{1} d s\right)^{+}\right]
$$


and

$$
x=S_{t} \quad \text { and } \quad y=\frac{1}{x}\left(\frac{1}{T} \int_{0}^{t} S_{u} d u-K\right) .
$$

As in the setting of European options, we may consider

$$
\text { Theta }_{t}=\frac{\partial C}{\partial t}(x, y, t, T) \quad \text { and } \quad \operatorname{Theta}_{T}=\frac{\partial C}{\partial T}(x, y, t, T),
$$

which however differ from each other in general, including when $\left(S_{t}\right)_{t \in \mathbb{R}_{+}}$is time homogeneous. Here, Theta $t_{t}$ can be computed from the Black-Scholes PDE for Asian options in a jump-diffusion model, cf. Proposition 9 of [1], as

$$
\begin{aligned}
\text { Theta }_{t}= & r C(x, y, t, T)+\left(\frac{1}{T}-a(y)\right) \frac{\partial C}{\partial y}(x, y, t, T)-\frac{1}{2} b^{2}(y) \frac{\partial^{2} C}{\partial y^{2}}(x, y, t, T) \\
& -(1+\eta) \lambda \int_{-\infty}^{+\infty}\left(C\left(x, \frac{y z}{1+\eta}, t, T\right)-C(x, y, t, T)\right) \nu(d z) .
\end{aligned}
$$

In the setting of Asian options, our method applies both to Theta $t$ and Theta $T$ and does not require the first and second derivatives $\frac{\partial C}{\partial y}$ and $\frac{\partial^{2} C}{\partial y^{2}}$. It also does not use Itô calculus.

Proposition 6.1 Given $u \in L^{2}([0, \tau])$ such that $\int_{0}^{\tau} D_{u} S_{s}^{x} d s \neq 0$, a.s., $s \in[0, \tau]$, let

$$
\Lambda(u)=-r-\frac{D_{u} S_{\tau}^{x}}{\int_{0}^{\tau} D_{u} S_{s}^{x} d s}+\frac{S_{\tau}^{x}-\int_{0}^{\tau} S_{s}^{x} d s / T}{\int_{0}^{\tau} D_{u} S_{s}^{x} d s}\left(I_{1}(u)+\frac{\int_{0}^{\tau} D_{u}^{2} S_{s}^{x} d s}{\int_{0}^{\tau} D_{u} S_{s}^{x} d s}\right),
$$

and assume that $\Lambda(u) \in L^{2}(\Omega)$. We have

$$
\operatorname{Theta}_{T}=x e^{-(T-t) r} \mathbb{E}\left[\Lambda(u)\left(y+\frac{1}{T} \int_{0}^{\tau} S_{s}^{1} d s\right)^{+}\right] .
$$

Proof. Replacing $x \mapsto(x-K)^{+}$in (6.1) by $\phi$ a $\mathcal{C}_{b}^{1}$ function we define the time derivative

$$
\begin{aligned}
\operatorname{Theta}_{T}^{\phi}= & \frac{\partial C}{\partial T}(x, y, t, T) \\
= & x e^{-(T-t) r} \mathbb{E}\left[-r \phi\left(y+\frac{1}{T} \int_{t}^{T} S_{s-t}^{1} d s\right)\right. \\
& \left.+\left(\frac{S_{T-t}^{1}}{T}-\frac{1}{T^{2}} \int_{t}^{T} S_{s-t}^{1} d s\right) \phi^{\prime}\left(y+\frac{1}{T} \int_{t}^{T} S_{s-t}^{1} d s\right)\right] .
\end{aligned}
$$

$\mathrm{RR} \mathrm{n}^{\circ} 5829$ 
Using (3.5) we have :

$$
\begin{aligned}
\operatorname{Theta}_{T}^{\phi}= & -r x e^{-(T-t) r} \mathbb{E}\left[\phi\left(y+\frac{1}{T} \int_{0}^{\tau} S_{s}^{1} d s\right)\right] \\
& +x e^{-(T-t) r} \mathbb{E}\left[\left(S_{\tau}^{1}-\frac{1}{T} \int_{0}^{\tau} S_{s}^{1} d s\right) \frac{D_{u} \phi\left(y+\int_{0}^{\tau} S_{s}^{1} d s / T\right)}{D_{u} \int_{0}^{\tau} S_{s}^{1} d s}\right] \\
= & -r x e^{-(T-t) r} \mathbb{E}\left[\phi\left(y+\frac{1}{T} \int_{0}^{\tau} S_{s}^{1} d s\right)\right] \\
& +x e^{-(T-t) r} \mathbb{E}\left[\left\langle\phi\left(y+\frac{1}{T} \int_{0}^{\tau} S_{s}^{1} d s\right), \frac{S_{\tau}^{1}-\int_{0}^{\tau} S_{s}^{1} d s / T}{\int_{0}^{\tau} D_{u} S_{s}^{1} d s} u\right\rangle\right] \\
= & -r x e^{-(T-t) r} \mathbb{E}\left[\phi\left(y+\frac{1}{T} \int_{0}^{\tau} S_{s}^{1} d s\right)\right] \\
& +x e^{-(T-t) r} \mathbb{E}\left[\phi\left(y+\frac{1}{T} \int_{0}^{\tau} S_{s}^{1} d s\right) \delta\left(\frac{S_{\tau}^{1}-\int_{0}^{\tau} S_{s}^{1} d s / T}{\int_{0}^{\tau} D_{u} S_{s}^{1} d s} u\right)\right] \\
= & x e^{-(T-t) r} \mathbb{E}\left[\phi ( y + \frac { 1 } { T } \int _ { 0 } ^ { \tau } S _ { s } ^ { 1 } d s ) \left(-r-\frac{D_{u} S_{\tau}^{1}}{\int_{0}^{\tau} D_{u} S_{s}^{1} d s}\right.\right. \\
& \left.\left.+\frac{S_{\tau}^{1}-\int_{0}^{\tau} S_{s}^{1} d s / T}{\int_{0}^{\tau} D_{u} S_{s}^{1} d s}\left(I_{1}(u)+\frac{\int_{0}^{\tau} D_{u}^{2} S_{s}^{1} d s}{\int_{0}^{\tau} D_{u} S_{s}^{1} d s}\right)\right)\right] \\
= & x e^{-(T-t) r} \mathbb{E}\left[\Lambda(u) \phi\left(y+\frac{1}{T} \int_{0}^{\tau} S_{s}^{1} d s\right)\right] .
\end{aligned}
$$

It remains to approximate $x \mapsto(x-K)^{+}$by a sequence $\left(\phi_{n}\right)_{n \in \mathbb{N}}$ of $\mathcal{C}_{b}^{1}$ functions.

Theta $_{t}$ can be computed in a similar way from

$$
C(x, y, t, T)=x e^{-(T-t) r} \mathbb{E}\left[\left(y+\frac{1}{T} \int_{0}^{T-t} S_{s}^{1} d s\right)^{+}\right]
$$

and

$\operatorname{Theta}_{t}^{\phi}=x e^{-(T-t) r} \mathbb{E}\left[r \phi\left(y+\frac{1}{T} \int_{0}^{T-t} S_{t-s}^{1} d s\right)+\frac{S_{T-t}^{1}}{T} \phi^{\prime}\left(y+\frac{1}{T} \int_{0}^{T-t} S_{s}^{1} d s\right)\right]$,

which yields after integration by parts:

$$
\text { Theta }_{t}=x e^{-(T-t) r} \mathbb{E}\left[\tilde{\Lambda}(u)\left(y+\frac{1}{T} \int_{0}^{T-t} S_{t-s}^{1} d s\right)^{+}\right],
$$


with

$$
\tilde{\Lambda}(u)=r-\frac{D_{u} S_{\tau}^{x}}{\int_{0}^{\tau} D_{u} S_{s}^{x} d s}+\frac{S_{\tau}^{x}}{\int_{0}^{\tau} D_{u} S_{s}^{x} d s}\left(I_{1}(u)+\frac{\int_{0}^{\tau} D_{u}^{2} S_{s}^{x} d s}{\int_{0}^{\tau} D_{u} S_{s}^{x} d s}\right) .
$$

In the geometric model (2.6) we have

$$
\Lambda(u)=-r-\frac{\int_{0}^{\tau} u_{s} d s S_{\tau}^{x}}{\int_{0}^{\tau} \int_{0}^{s} u_{p} d p S_{s}^{x} d s}+\frac{S_{\tau}^{x}-\int_{0}^{\tau} S_{s}^{x} d s / T}{\sigma \int_{0}^{\tau} \int_{0}^{s} u_{p} d p S_{s}^{x} d s}\left(I_{1}(u)+\sigma \frac{\int_{0}^{\tau}\left(\int_{0}^{s} u_{p} d p\right)^{2} S_{s}^{x} d s}{\int_{0}^{\tau} \int_{0}^{s} u_{p} d p S_{s}^{x} d s}\right)
$$

and

$$
\tilde{\Lambda}(u)=r-\frac{\int_{0}^{\tau} u_{s} d s S_{\tau}^{x}}{\int_{0}^{\tau} \int_{0}^{s} u_{p} d p S_{s}^{x} d s}+\frac{S_{\tau}^{x}}{\sigma \int_{0}^{\tau} \int_{0}^{s} u_{p} d p S_{s}^{x} d s}\left(I_{1}(u)+\sigma \frac{\int_{0}^{\tau}\left(\int_{0}^{s} u_{p} d p\right)^{2} S_{s}^{x} d s}{\int_{0}^{\tau} \int_{0}^{s} u_{p} d p S_{s}^{x} d s}\right) .
$$

\section{$7 \quad$ Numerical simulations}

In this section we consider the geometric Brownian model

$$
\left\{\begin{array}{l}
d S_{s}=r S_{s} d s+\sigma S_{s} d W_{s} \\
S_{t}=x
\end{array}\right.
$$

and apply the Malliavin formula (5.1) with $\tilde{u}_{s}=\tilde{v}_{s}=\tilde{w}_{s}=1 / \tau, s \in[0, \tau]$, to compute

$$
\text { Theta }_{T}=- \text { Theta }_{t}
$$

for European type options with non-smooth payoff functions. Formula (4.1) is used for finite differences approximations.

\subsection{Digital options}

In case $\phi=\mathbf{1}_{[K,+\infty)}$, the value of Theta can be computed analytically as

$$
\text { Theta }=e^{-r \tau}\left(e^{-\frac{a^{2}}{2 \tau}}\left(\frac{r}{\sigma}-\frac{\sigma}{2}+\frac{a}{2 \tau \sqrt{2 \pi \tau}}\right)-r \int_{\frac{a}{\sqrt{\tau}}}^{\infty} \frac{e^{-\frac{y^{2}}{2}}}{\sqrt{2 \pi}} d y\right),
$$

with $a=(\ln (K / x)-r \tau) / \sigma$, cf. e.g. [8], which yields the following graph:

$\mathrm{RR} \mathrm{n}^{\circ} 5829$ 


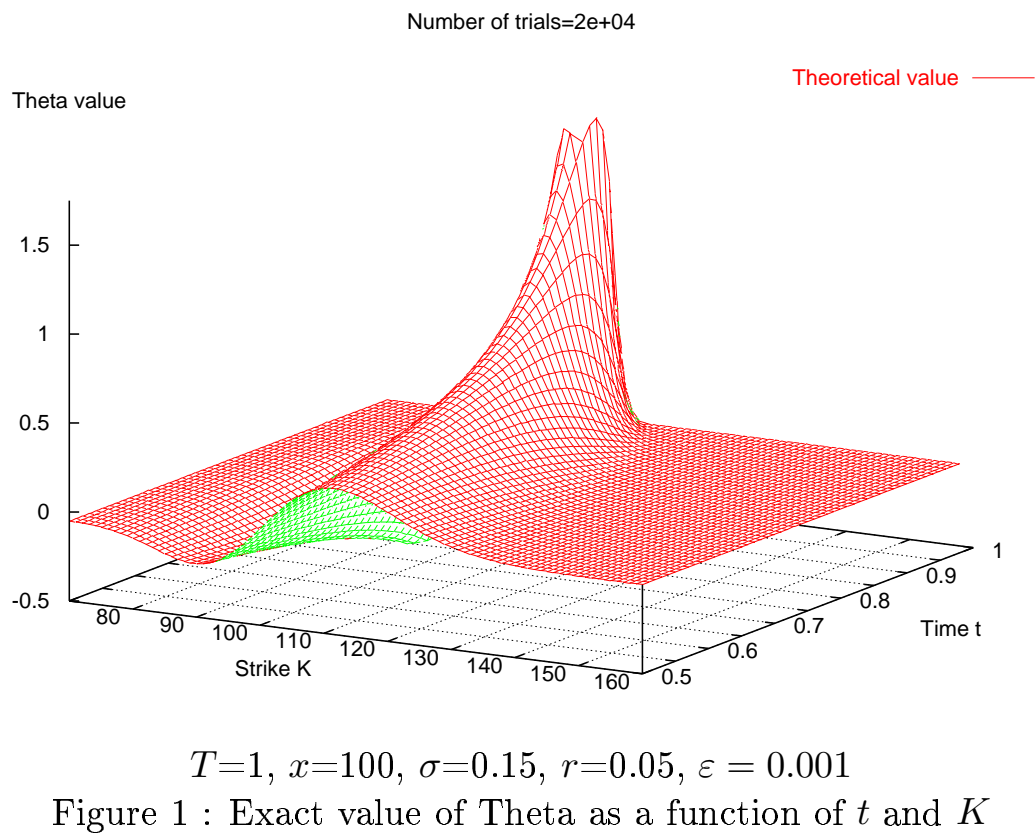

The next graphs allow us to compare the Monte Carlo simulations obtained by finite differences and integration by parts, first in terms of number of samples and then as functions of $t$ and $K$. 


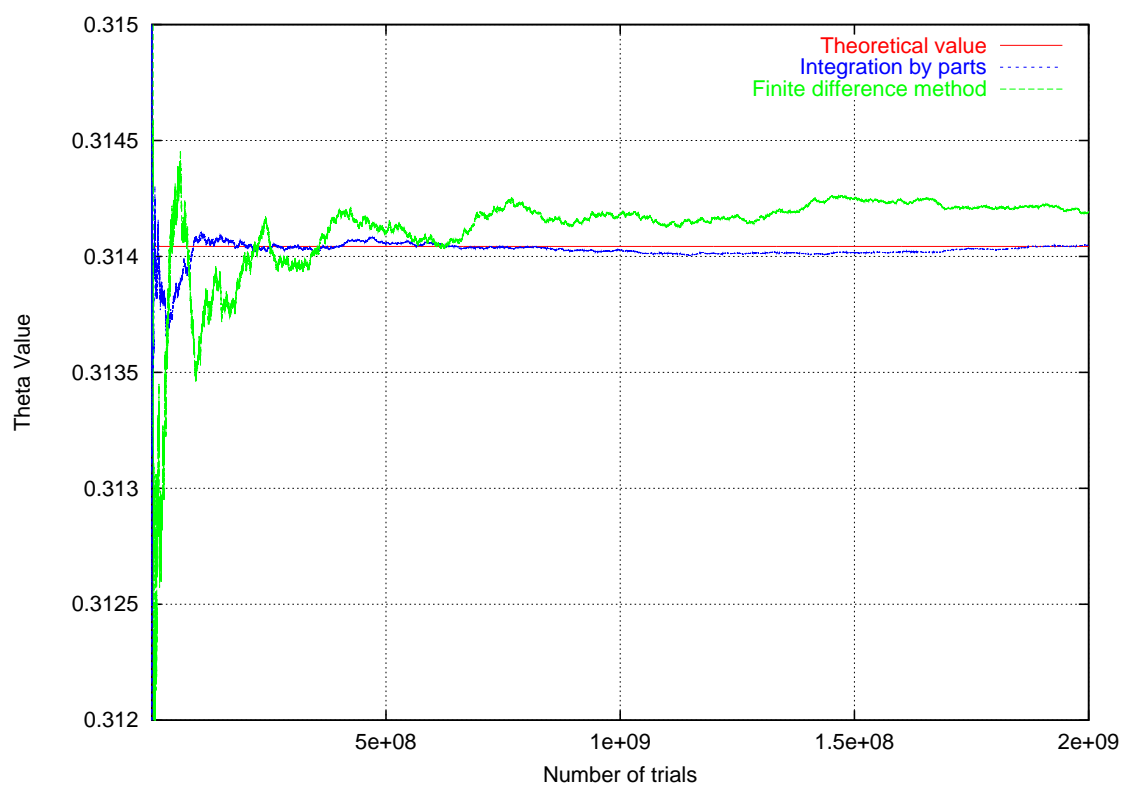

$T=1, t=0.5, x=100, \sigma=0.2, r=0.1, K=110, \varepsilon=0.01$

Figure 2 : Estimation of Theta vs number of trials

Number of trials $=2 e+04$

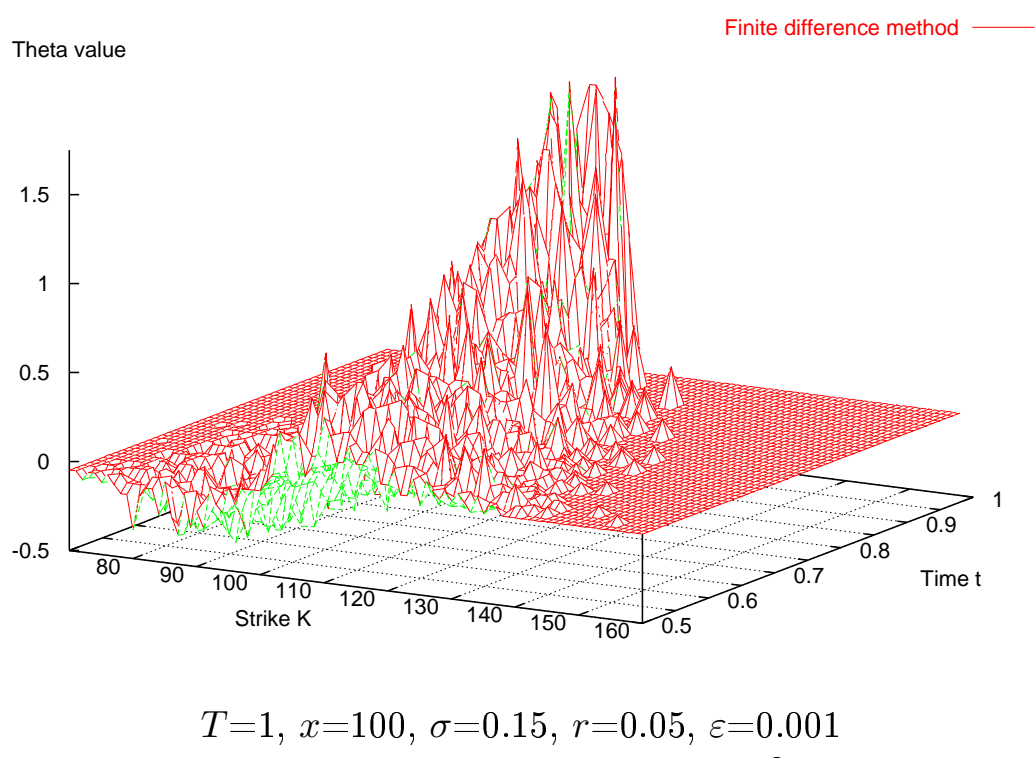

Figure 3 : Theta as a function of $t$ and $K$ by finite differences

$\mathrm{RR} \mathrm{n}^{\circ} 5829$ 


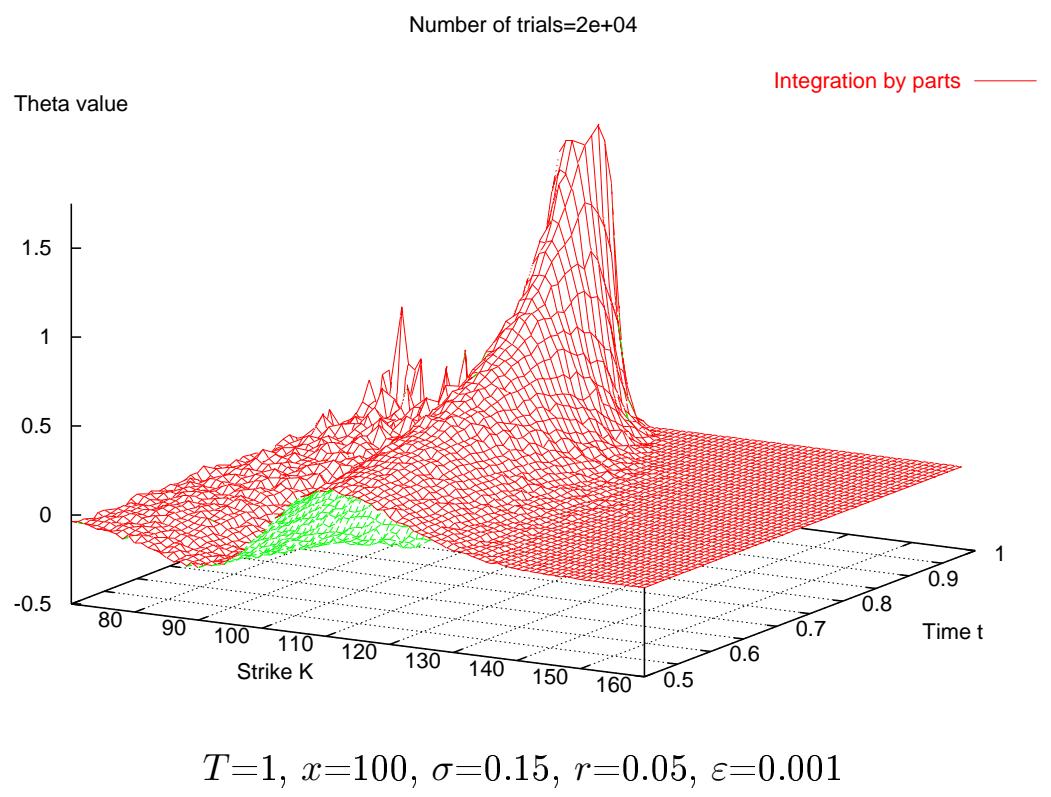

Figure 4 : Theta as a function of $t$ and $K$ by integration by parts

\subsection{European options}

In case $\phi(x)=(x-K)^{+}$, Theta can be computed as

$$
\text { Theta }=\frac{x \sigma}{2 \sqrt{2 \pi \tau}} e^{-c^{2} / 2}+K r e^{-r \tau} N(c-\sigma \sqrt{\tau}),
$$

where $c=\left(\sigma-\frac{a}{\tau}\right) \sqrt{\tau}$ and $N(x)=\int_{-\infty}^{x} e^{-\frac{y^{2}}{2}} \frac{d y}{\sqrt{2 \pi}}$, cf. e.g. [8], which yields the following graph. 


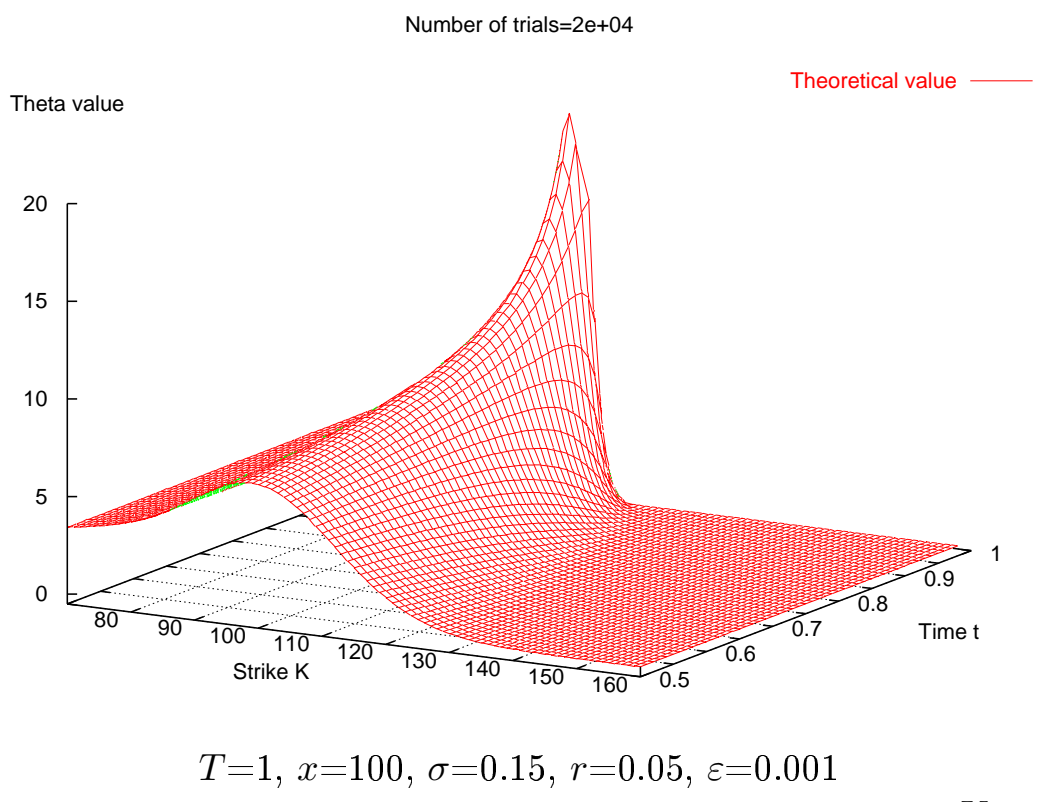

Figure 5 : Exact value of Theta as a function of $t$ and $K$

As in [4], the following graphs show that the finite difference approximation performs better for European calls with $\phi(x)=(x-K)^{+}$, but as seen in the previous section, the Malliavin calculus approach performs better for digital options.

$\mathrm{RR} \mathrm{n}^{\circ} 5829$ 
Number of trials $=2 e+04$

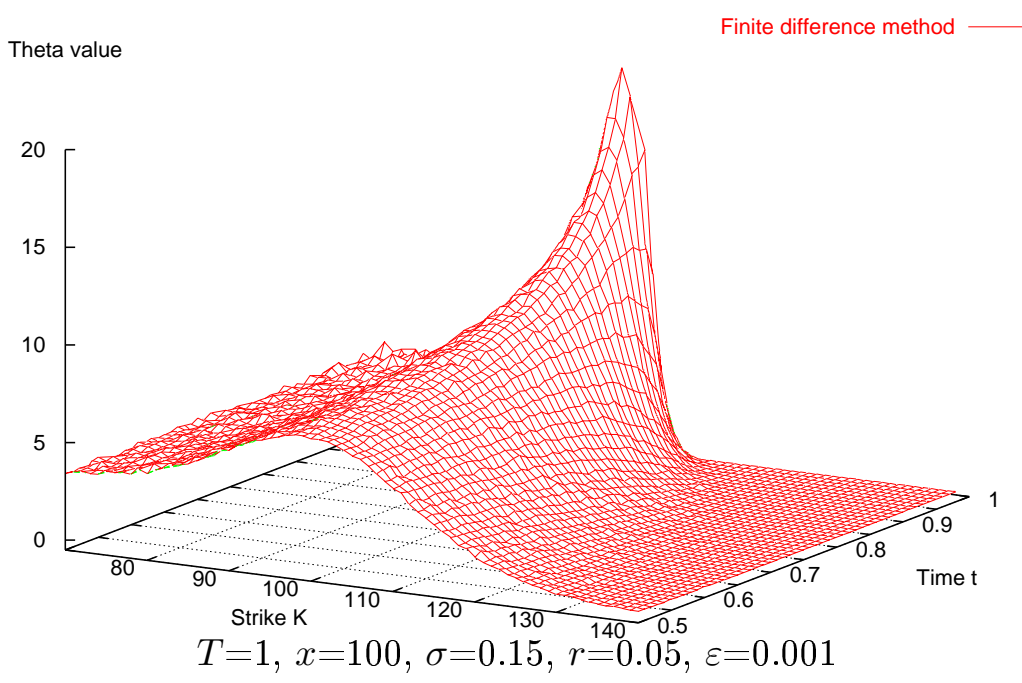

Figure 6 : Theta as a function of $t$ and $K$ by finite differences

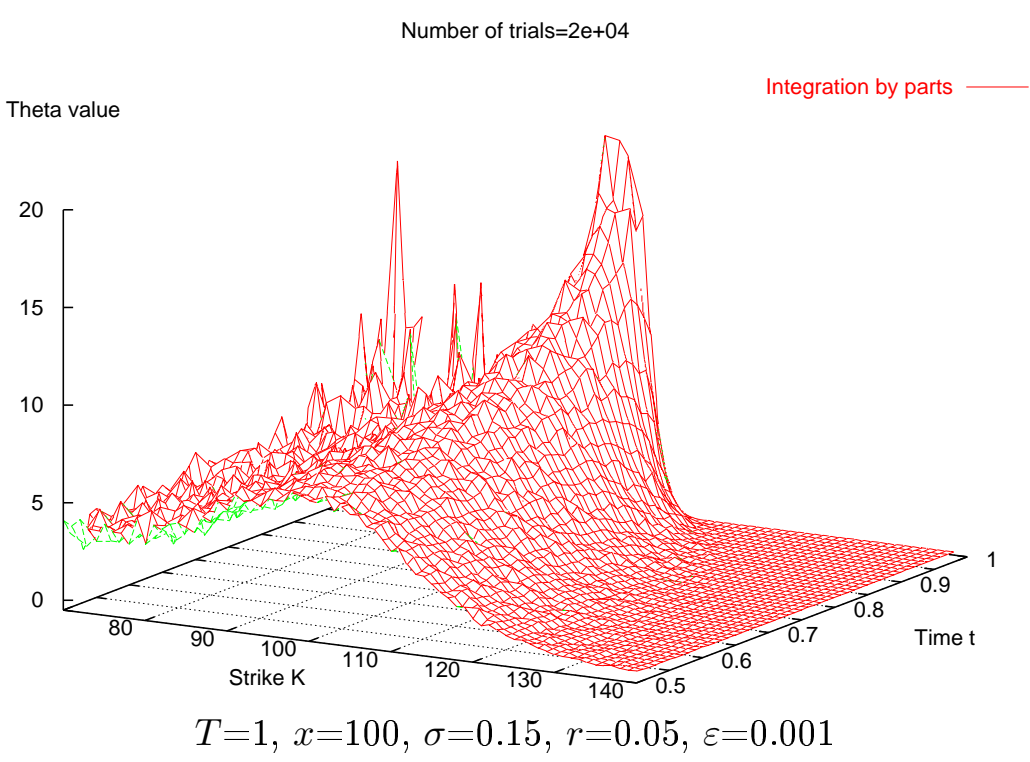

Figure 7 : Theta as a function of $t$ and $K$ by integration by parts

The localization procedure applied in the next section will allow us to improve the result of the Malliavin method. 


\section{Localization}

Payoff functions of the form

$$
\phi(y)=\mathbf{1}_{[K,+\infty)}(y) \quad \text { and } \quad \phi(y)=(y-K)^{+}
$$

have a singularity at $y=K$. The idea of localization is to decompose the payoff function $\phi$ as

$$
\phi=g_{\varepsilon}+h_{\varepsilon}
$$

in such a way that $h_{\varepsilon}$ is twice differentiable and $g_{\varepsilon}$ contains the singularity of $\phi$, see e.g. [9] for digital options and [2] for European options. Applying the Malliavin approach to $g_{\varepsilon}$ and using (4.3) we get

$$
\begin{aligned}
\text { Theta }= & e^{-\tau r} \mathbb{E}\left[\left(\Lambda(u, v, w) g_{\varepsilon}\left(S_{\tau}^{x}\right)+\lambda \int_{-\infty}^{+\infty}\left(\phi\left(S_{\tau}^{x}+c\left(S_{\tau}^{x}\right) y\right)-\phi\left(S_{\tau}^{x}\right)\right) \nu(d y)\right)\right] \\
& -r e^{-\tau r} \mathbb{E}\left[h_{\varepsilon}\left(S_{\tau}^{x}\right)\right]+e^{-\tau r} \mathbb{E}\left[h_{\varepsilon}^{\prime}\left(S_{\tau}^{x}\right) a\left(S_{\tau}^{x}\right)\right] \\
& +\frac{1}{2} e^{-\tau r} \mathbb{E}\left[h_{\varepsilon}^{\prime \prime}\left(S_{\tau}^{x}\right) b^{2}\left(S_{\tau}^{x}\right)\right] .
\end{aligned}
$$

We applied the integration by parts method to get rid of the first and second derivatives on $g_{\varepsilon}$. In the geometric model with the optimal weight $\Lambda(u, v, w)$ this gives:

$$
\begin{aligned}
\text { Theta }= & -r e^{-r \tau} \mathbb{E}\left[\phi\left(S_{\tau}^{x}\right)\right] \\
& +r \frac{e^{-r \tau}}{\sigma \tau} \mathbb{E}\left[g_{\varepsilon}\left(S_{\tau}^{x}\right) W_{\tau}\right]+\frac{e^{-r \tau}}{2 \tau} \mathbb{E}\left[g_{\varepsilon}\left(S_{\tau}^{x}\right)\left(\frac{W_{\tau}^{2}}{\tau}-\sigma W_{\tau}-1\right)\right] \\
& +r e^{-r \tau} \mathbb{E}\left[S_{\tau}^{x} h_{\varepsilon}^{\prime}\left(S_{\tau}^{x}\right)\right]+\frac{\sigma^{2}}{2} e^{-r \tau} \mathbb{E}\left[S_{\tau}^{x 2} h_{\varepsilon}^{\prime \prime}\left(S_{\tau}^{x}\right)\right]
\end{aligned}
$$

For digital options we take

$$
\begin{aligned}
h_{\varepsilon}(y) & =\frac{1}{2}\left(1+\frac{y-K}{\varepsilon}\right)^{2} \mathbf{1}_{]-\varepsilon, 0]}(y-K)+\left(1-\frac{1}{2}\left(1-\frac{y-k}{\varepsilon}\right)^{2}\right) \mathbf{1}_{] 0, \varepsilon[}(y-K) \\
& +\mathbf{1}_{[\varepsilon,+\infty)}(y-K),
\end{aligned}
$$

and obtain the following graphs by Monte-Carlo simulation:

$\mathrm{RR} \mathrm{n}^{\circ} 5829$ 


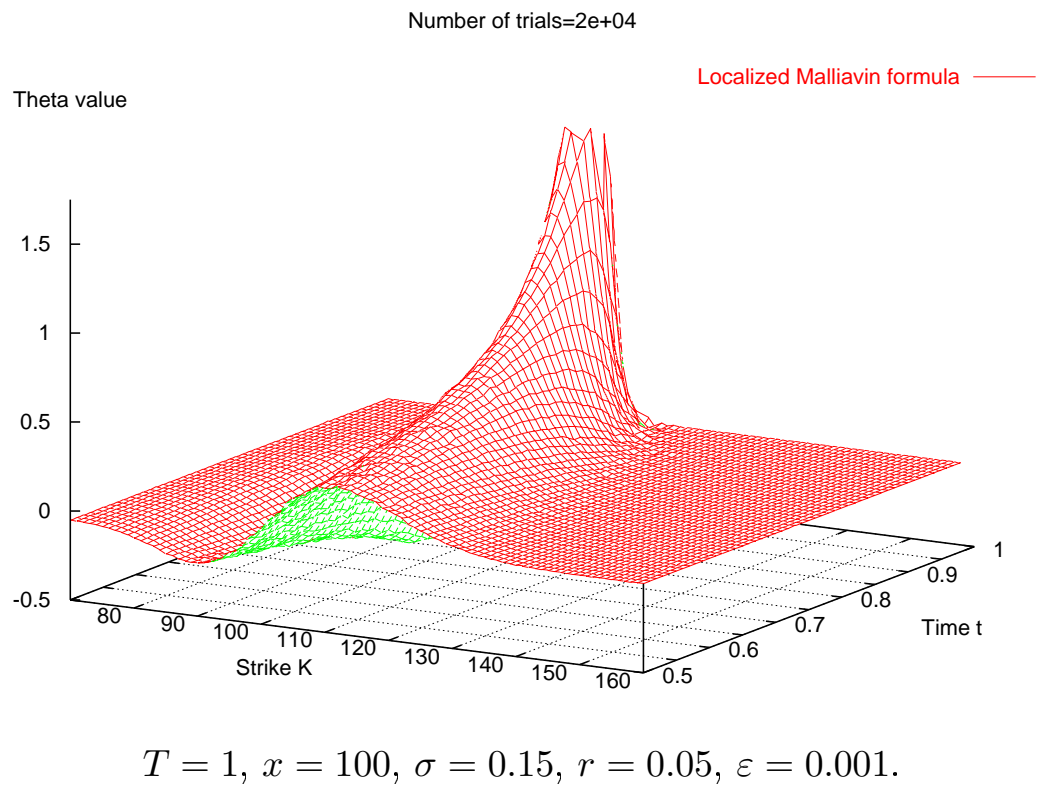

Figure 8 : Theta as a function of $t$ and $K$ by the localized Malliavin method

In the case of European options we take

$$
h_{\varepsilon}(y)=\frac{1}{4 \varepsilon}(y-(K-\varepsilon))^{2} \mathbf{1}_{[-\varepsilon, \varepsilon]}(y-K)+(y-K) \mathbf{1}_{] \varepsilon,+\infty)}(y-K),
$$

which yields the following graph by Monte Carlo simulation: 


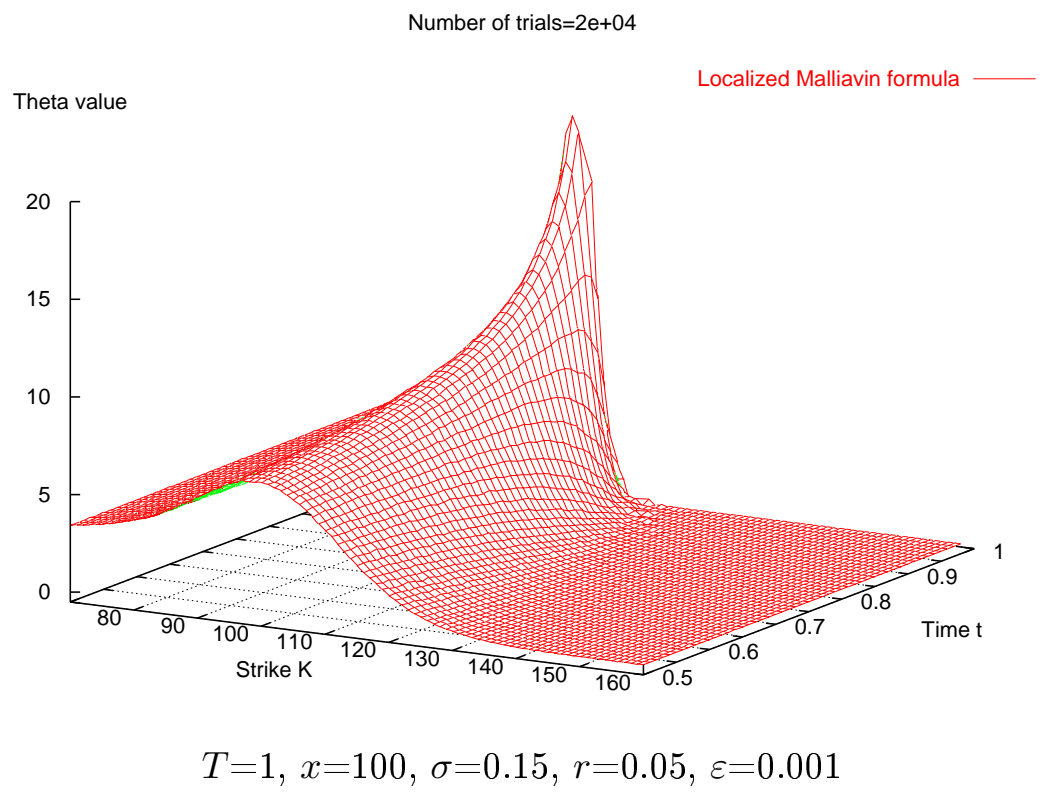

Figure 9 : Theta as a function of $t$ and $K$ by the localized Malliavin method

\section{References}

[1] N. Bellamy. Asian options in a market driven by a discontinuous process. Finance, 20(2):69-93, 1999.

[2] F.E. Benth, L.O. Dahl, and K.H. Karlsen. Quasi Monte-Carlo evaluation of sensitivities of options in commodity and energy markets. Int. J. Theor. Appl. Finance, 6(8):865-884, 2003.

[3] R. Cont and P. Tankov. Financial modelling with jump processes. Chapman \& Hall/CRC Financial Mathematics Series. Chapman \& Hall/CRC, Boca Raton, FL, 2004.

[4] M.H.A. Davis and M.P. Johansson. Malliavin Monte Carlo Greeks for jump diffusions. Stochastic Processes and their Applications, 116(1):101-129, 2006.

[5] V. Debelley and N. Privault. Sensitivity analysis of European options in jump diffusion models via the Malliavin calculus on Wiener space. Preprint, 2004.

[6] B. Dupire. Pricing with a smile. Risk, 7(1):18-20, 1994.

[7] E. Fournié, J.M. Lasry, J. Lebuchoux, P.L. Lions, and N. Touzi. Applications of Malliavin calculus to Monte Carlo methods in finance. Finance and Stochastics, 3(4):391-412, 1999.

[8] M. Kijima. Stochastic processes with applications to finance. Chapman \& Hall/CRC, Boca Raton, FL, 2003.

[9] L. Nguyen. Application du calcul de Malliavin à la finance. Master's thesis, Université de Paris $6,1999$.

$\mathrm{RR} \mathrm{n}^{\circ} 5829$ 
[10] D. Nualart. The Malliavin Calculus and Related Topics. Probability and its Applications. Springer-Verlag, 1995.

[11] L.C.G. Rogers and Z. Shi. The value of an asian option. J. Appl. Probab., 32(4):1077-1088, 1995. 


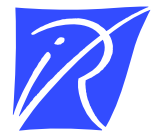

Unité de recherche INRIA Rocquencourt

Domaine de Voluceau - Rocquencourt - BP 105 - 78153 Le Chesnay Cedex (France)

Unité de recherche INRIA Futurs : Parc Club Orsay Université - ZAC des Vignes

4, rue Jacques Monod - 91893 ORSAY Cedex (France)

Unité de recherche INRIA Lorraine : LORIA, Technopôle de Nancy-Brabois - Campus scientifique

615, rue du Jardin Botanique - BP 101 - 54602 Villers-lès-Nancy Cedex (France)

Unité de recherche INRIA Rennes : IRISA, Campus universitaire de Beaulieu - 35042 Rennes Cedex (France)

Unité de recherche INRIA Rhône-Alpes : 655, avenue de l'Europe - 38334 Montbonnot Saint-Ismier (France)

Unité de recherche INRIA Sophia Antipolis : 2004, route des Lucioles - BP 93 - 06902 Sophia Antipolis Cedex (France)

INRIA - Domaine de Voluceau - Rocquencourt, BP 105 - 78153 Le Chesnay Cedex (France)

http://www.inria.fr

ISSN 0249-6399 